\title{
Characterisation of Fusion Bonding between Filaments of Thin 3D Printed Polyamide 6 Using an Essential Work of Fracture Method
}

\author{
Qinghao He a, Lin Ye ${ }^{\mathrm{a},{ }^{*}}$, Anthony J. Kinloch ${ }^{\mathrm{a}, \mathrm{b}}$, Hongjian Wang ${ }^{\mathrm{a}}$, and Binghong Yin ${ }^{\mathrm{a}}$ \\ ${ }^{\text {a }}$ Centre for Advanced Materials Technology, School of Aerospace, Mechanical and Mechatronic Engineering, The \\ University of Sydney, NSW 2006, Australia \\ b Department of Mechanical Engineering, Imperial College London, South Kensington Campus, London SW7 2AZ, \\ UK
}

*E-mail address: lin.ye@sydney.edu.au

\begin{abstract}
The extent and quality, i.e. consistency, of the fusion bonding between filaments that occurs during the fused filament fabrication (FFF) process plays an important role in determining the mechanical performance of 3D printed polymeric parts. This present research is designed to quantify these parameters of the fusion bonding between individual filaments deposited during the 3D printing process. Polyamide 6 (PA6) specimens were prepared from both 3D printed single-layer and single-filament films. Also, to provide a benchmark material, 3D printed PA6 films prepared by the FFF process were further processed by using compression moulding (CM) to investigate whether the fusion bonding could be enhanced. An essential work of fracture (EWF) method was employed to evaluate the fusion bonding between the filaments by determining the value of the specific essential work of fracture, $w_{e}$, and the associated experimental reproducibility. The higher value of $w_{e}$, and the relatively low degree of scatter, determined for the benchmark material revealed that the extent and quality of fusion bonding attained when using only the FFF process clearly needed to be improved when parts are fabricated for practical applications. To study the effect of temperature on the fusion bonding, the structural detail and fracture surfaces of thin polymer films were studied, with the thermal history of the deposited filaments also being measured using a thermal camera.
\end{abstract}

Keywords: 3D printing; Fusion bonding; Adhesion; Essential work of fracture; Fused filament fabrication. 


\section{Introduction}

The mechanical performance of 3D printed parts produced by the fused filament fabrication (FFF) process, where the bulk polymeric material part is built layer by layer from the deposition of fused polymeric filaments, is highly dependent on the extent and quality of the fusion bonding between the deposited filaments. Rodríguez et al. [1] reported that materials fabricated using an acrylonitrile butadiene styrene (ABS) polymer by the FFF process suffered from a loss of elastic modulus and strength (of up to $37 \%$ and $57 \%$, respectively) compared with the feedstock material, i.e. the monofilament of the ABS polymer, and that these effects could be attributed to the presence of voids plus loss of molecular orientation. Several other researchers [2-5] have tried to obtain improved mechanical properties by optimising the processing parameters of the FFF process such as the printing orientation, layer thickness, raster angle, nozzle temperature, ambient temperature, and so on. Besides the optimisation of the printing parameters, the development of new raw materials for FFF by adding reinforcements to the materials has also attracted intense interest.

Some studies have evaluated the extent of fusion bonding within a printed structure produced by the FFF process and when an insufficient bonding strength was achieved between filaments this has been found to have a negative impact on the mechanical properties. For instance, Sun et al. [6] reported the effects of the envelope temperature and convective conditions on the fusion bonding between adjacent filaments and the resulting flexural properties, where the quality of the fusion bonding was quantified using the radius of neck growth at the interface. A follow-up study by Abbott et al. [7] investigated the relationship between selected print parameters, such as thermal history, mesostructure and tensile strength, and the contact length of the coalesce formed between individual filaments, which was measured using optical microscopy. This enabled these research workers to characterise the in-plane and interlayer bonding strength. Yin et al. [8] studied the interfacial bonding between a thermoplastic polyurethane (TPU) and an ABS polymer based on the tensile strength evaluated by uniaxial tensile testing of cuboid specimens. Thus, so far, the characterisation of the extent of fusion bonding has relied mainly on either mesostructural analysis or mechanical tests on printed bulk specimens consisting of multiple layers. However, an evaluation of the fusion bonding between individual filaments in 3D printing by the FFF process would give more insights into the quality of the coalescence between the filaments, which occurs via a mechanism frequently referred to as 'molecular inter- 
diffusion'. In fact, evaluation of the fusion bonding between individual filaments in 3D printing is critical for the comprehensive understanding of the FFF process and would certainly pave the way for future process optimisation.

With this background in mind, the essential work of fracture (EWF) method, widely accepted for determining the toughness of ductile polymers [9], was adopted in this study to investigate firstly the fusion bonding, i.e. the adhesion via molecular inter-diffusion, in both the 'in-plane' and 'in-thickness' deposition modes of filaments of polyamide 6 (PA6). This EWF test method involves measuring the specific fracture energy of 3D printed thin films under a plane-stress condition using double edge-notched tension (DENT) specimens. Secondly, EWF tests were also performed on 3D printed PA6 films which had been subjected to further compression moulding (CM) to promote further molecular inter-diffusion. The aim here is to identify any differences in the extent of fusion bonding and material mesostructures developed during the two types of fusion processes. Finally, in order to identify the process-structure-property relationships, measurements of the thermal history during the FFF process and of the nano-mechanical properties were also conducted.

\section{Materials and experimental setup}

\subsection{Materials and specimen preparation}

To characterise the fusion bonding of 3D printed PA6 using the FFF process, specimens were fabricated using a desktop 3D printer (Markforged ${ }^{\circledR}$ Mark Two, USA) using proprietary Tough Nylon ${ }^{\circledR}$ feedstock material. The diameter of the extruded filaments of PA6 before deposition was $0.4 \mathrm{~mm}$, which was about the same size as the printing nozzle, and the printing temperature was $275^{\circ} \mathrm{C}$. Firstly, to characterise the fusion bonding of 3D printed PA6 filaments deposited by an 'in-plane' mode during the FFF process, singlelayer thin films, $100 \times 85 \mathrm{~mm}$ in size, were fabricated, as shown in Figure 1(a). It should be noted that the nominal thickness of this single-layer film is about $0.125 \mathrm{~mm}$, due to the extruded filaments of the PA6 being flattened by the pressure associated with the printing process, as discussed later. Secondly, to characterise the fusion bonding of 3D printed PA6 filaments deposited by an 'in-thickness' mode during the FFF process, a box structure (Figure 1(b)) was designed and fabricated using the PA6 feedstock material, with a nominal thickness of $0.4 \mathrm{~mm}$ for each side wall and being $110 \times 110 \mathrm{~mm}$ in cross-section and $90 \mathrm{~mm}$ in height. The 
walls of the fabricated box provided 'in-thickness' deposition mode specimens, since here a single filament was deposited on top of another single filament during the FFF process. In Figures 1(a) and 1(b) the orientations of the PA6 filaments in the double edge-notched tension (DENT) specimens used to measure the fracture toughness, and hence assess the extent and quality of fusion bonding between the filaments, for the ‘in-plane' and 'in-thickness' deposition modes are also indicated, as labelled by 'S1' and S2'. Thirdly, to provide a benchmark material for this work, PA6 films with a nominal thickness of 0.25 mm were first 3D printed by the 'in-plane' deposition mode, and then hot pressed using CM to ensure thorough and uniform molecular inter-diffusion. This was undertaken using a steel mould and, after the 3D printed PA6 films had stabilised at the desired temperature of $220^{\circ} \mathrm{C}$, a pressure of $2 \mathrm{MPa}$ was applied and held for 5 minutes, followed by cooling to room temperature.

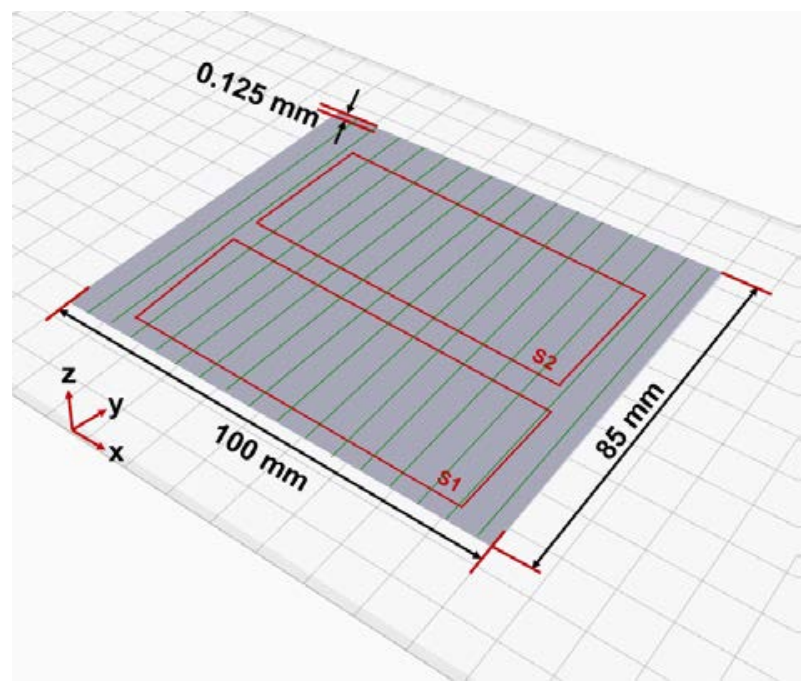

(a)

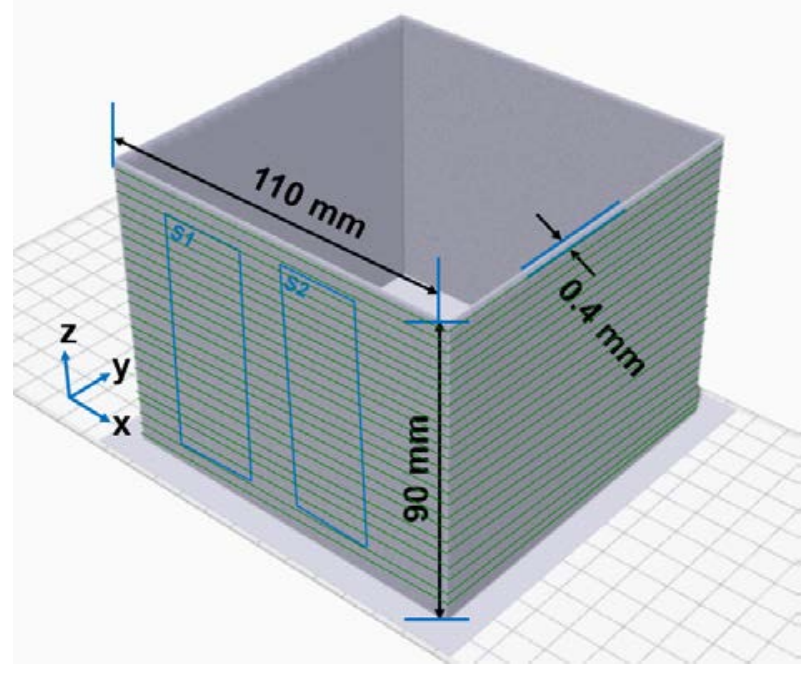

(b)

Figure 1. Schematic of the 3D printed DENT specimens with the orientation of the deposited PA6 filaments shown (as the green lines): (a) a single-layer film printed in the $x-y$ plane for 'in-plane' deposition mode tests and (b) a single-filament box in 3D printed for 'in-thickness' deposition mode tests. The orientations of the double edge-notched tension (DENT) specimens, labelled 'S1' and 'S2', used to assess the extent of fusion bonding are also indicated.

Figure 2(a) shows a schematic of the DENT specimen with a width, $W$, of $30 \mathrm{~mm}$ and a height, $H$, of $80 \mathrm{~mm}$, and with a ligament length, $L$. Two DENT 'in-plane' deposition mode specimens could be cut from a 
thin single-layer film printed in the x-y plane by a laser cutter (Epilog Fusion M2), as indicated by the two rectangles, labelled 'S1' and 'S2', shown in Figure 1(a). By locating the specimens within the printed films as centrally as possible, the edge effects resulting from the printing process were minimised. Similarly, two specimens for the 'in-thickness' deposition mode tests were obtained from each side of the printed square box. In addition, the benchmark DENT specimens were cut from 3D printed 'in-plane' thin films with further CM, as described above. For each DENT specimen, both edges were notched by a slitting motion of a sharp razor blade to obtain a ligament with the nominal length, $L$, ranging from $2 \mathrm{~mm}$ to $10 \mathrm{~mm}$ at approximately equally spaced intervals. For the DENT specimens cut from the same printed film, the ligament length was made different to avoid a batch effect which could be caused by various environmental conditions or the calibration status of the printing machine, etc.

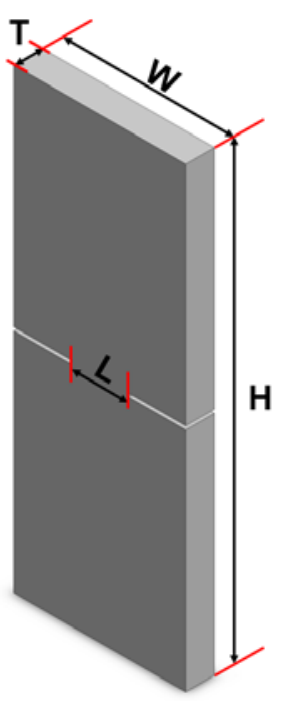

(a)

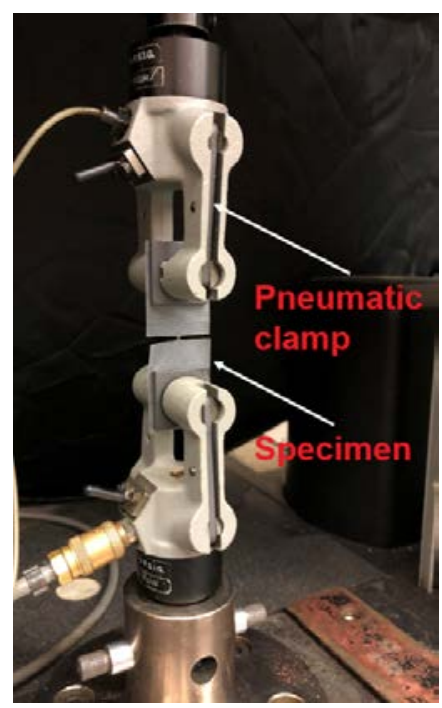

(b)

Figure 2. (a) A schematic of the double edge-notched tension (DENT) specimen and (b) the essential work of fracture (EWF) test.

\subsection{The EWF test}

The EWF test method has been shown to be applicable to ductile polymers [9-13] providing the following criteria are met: (a) full ligament yielding occurs prior to crack initiation, (b) self-similar loaddisplacement curves are measured, (c) plane-stress conditions prevail and (d) the volume of the outer plastic 
dissipation zone approximately scales with the square of the ligament. As shown below, these conditions are all met for the current EWF tests and, thus, in accordance with the theory of EWF, the total energy dissipated during an EWF test is:

$$
W_{f}=\int_{0}^{\delta_{C}} P d \delta
$$

where $W_{f}$ is the total work of fracture, $P$ is the tensile load applied to the specimen and $\delta$ is the corresponding measured displacement until complete failure occurs. In principle, the term $W_{f}$ can be divided into two parts, the essential work done in tearing the ligament, $W_{e}$, and the plastic work done in a more diffuse zone, $W_{p}[10]$. For plane-stress conditions, $W_{e}$ is proportional to the length of the ligament, $L$, while $W_{p}$ is proportional to $L^{2}$ [11], and thus Equation 1 can be written as:

$$
W_{f}=W_{e}+W_{p}=w_{e} L T+\beta w_{p} L^{2} T
$$

where $w_{e}$ is the specific essential work of fracture, $w_{p}$ is the specific non-essential work, $T$ is the thickness of the DENT specimen, and $\beta$ is a shape factor. Hence, if both sides of Equation 2 are divided by the area of the ligament region (i.e. $L \times T$ ), the relationship between the specific total work of fracture, $w_{f}$, and the liagment length, $L$, will be:

$$
w_{f}=w_{e}+\beta w_{p} L
$$

Thus, if several specimens with different ligament lengths are tested, $w_{f}$ can be plotted against $L$, where the positive intercept of the least squares regression line would be $w_{e}$, with the slope being $\beta w_{p}$. The value of $w_{e}$ so obtained was used to quantify the toughness of all the polymer films fabricated in this study and, more importantly, to evaluate the extent of fusion bonding developed during the FFF process. Finally, it should be noted, that although the three different types of DENT specimens have somewhat different thicknesses, the calculated values of the the specific essential work of fracture, $w_{e}$, have been shown $[12,13]$ to be independent of the thickness, providing the specimens are relatively thin and plane-stress conditions are present, as is the case in the present tests.

In the linear regression analysis, the coefficient of determination, $\mathrm{R}^{2}$, can be determined, which describes how the variation of the specific work of fracture, $w_{f}$, is directly related to the change in ligament length, $L$ : 


$$
\mathrm{R}^{2}=\left[\frac{1}{n-1} \sum_{i=1}^{n}\left(\frac{L_{i}-\bar{L}}{S_{L}}\right)\left(\frac{w_{f_{i}}-\overline{w_{f}}}{S_{w_{f}}}\right)\right]^{2}
$$

where $n$ is the total number of EWF tests, $w_{f_{i}}$ and $L_{i}$ represent the specific work of fracture and the ligament length, respectively, from the $i$-th test and $\overline{w_{f}}$ and $\bar{L}$ are their mean values, respectively, and $S_{w_{f}}$ and $S_{L}$ are their standard deviations, respectively. Ideally, for a large number of EWF tests on a material of perfect consistency, without any experimental error, the value of $\mathrm{R}^{2}$ is 1.0 . Hence, for the current EWF tests, a relatively low value of $\mathrm{R}^{2}$ suggests an inconsistent quality of the tested material.

\subsection{Experimental setup}

To check the crystallinity of the 3D printed PA6 films, differential scanning calorimetry (DSC) tests were run using a 2920 Modulated DSC (TA instruments Co., Ltd., USA). The samples were encapsulated in an aluminium pan and heated at the rate of $10^{\circ} \mathrm{C} / \mathrm{min}$ from 30 to $300{ }^{\circ} \mathrm{C}$ in a nitrogen atmosphere, with the heat flow recorded during the test. Fourier transform infrared spectroscopy (FTIR, Agilent 4300) analysis was performed on the PA6 films before and after CM with a diamond attenuated total reflectance interface, using a resolution of $4 \mathrm{~cm}^{-1}$ and sixty four scans, to detect any changes in the chemical composition of the PA6 polymer due to the further use of the CM process.

Before the EWF tests were conducted, the ligament length of each DENT specimen was measured using an optical microscope. Figure 3(a) shows an optical micrograph of a 3D printed specimen for the 'inplane' deposition mode with a nominal ligament length of $3 \mathrm{~mm}$, and which illustrates that the actual ligament length can be measured with a high degree of accuracy. The crack tip profile of a notched DENT specimen was checked using scanning electron microscopy (SEM), as shown in Figure 3(b). The crack tip generated by the razor blade is very sharp with the tip radius being much smaller than $15 \mu \mathrm{m}$, which represents a threshold value for valid EWF tests [10]. The thickness of each specimen was measured three times using a precision micrometer, with the average value being used to define the thickness, $T$, in Equation 2. 


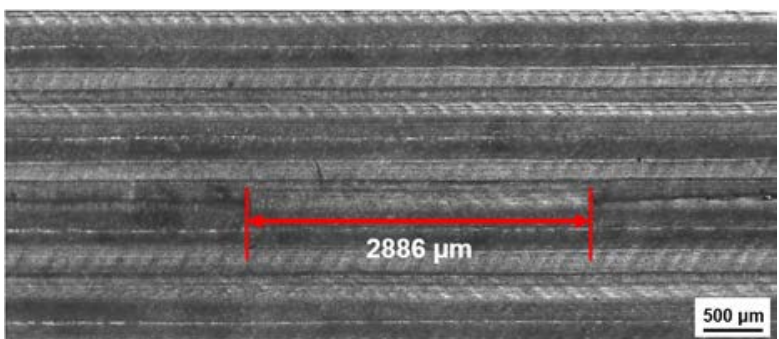

(a)
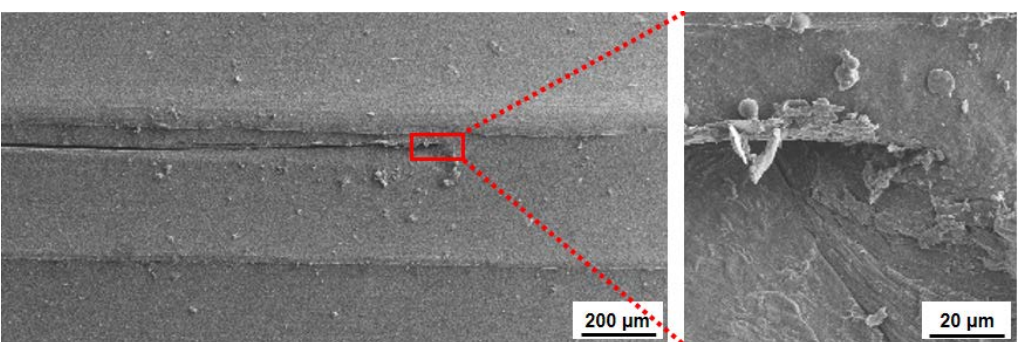

(b)

Figure 3. Micrographs: (a) optical measurement of a nominal 3 mm long ligament of a PA6 DENT specimen and (b) SEM images of the sharp crack tip generated by a razor blade.

EWF tests were carried out using a universal testing machine (Instron 5567) equipped with a $100 \mathrm{~N}$ load cell at the quasi-static speed of $2 \mathrm{~mm} / \mathrm{min}$, as shown in Figure 2(b). To avoid any slippage between the thin specimen and the pneumatic clamping fixture, pieces of sandpaper of an appropriate size were used to ensure that firm clamping was achieved. A pre-load of $\sim 0.2 \mathrm{~N}$ was applied to obtain an appropriate alignment of the specimen. All EWF tests were video-recorded using a high-resolution camera, simultaneously activated at the start of an EWF test, to observe the fracture behaviour, e.g. the blunting of cracks, crack propagation, etc. No fewer than twenty-five specimens were tested for each different type of EWF test, i.e. for the 'in-plane' and 'in-thickness' deposition mode specimens, and for the benchmark 'in-plane' deposition mode with further CM specimens, to ensure an accurate determination of $w_{e}$. The test environment was controlled to give a nominal relative humidity of $60 \%$ and a nominal room temperature of $25{ }^{\circ} \mathrm{C}$.

Several polished cross-sections of the DENT specimens before and after the EWF test were observed using an optical microscope, with the thickness measured by using image-processing software. For a better understanding of the process-structure-property relationships, fracture surfaces of the DENT specimens were also observed after testing using SEM. An accelerating voltage of $5 \mathrm{kV}$ was used for the SEM imaging. Additionally, the temperature-time response of the PA6 during the FFF process was recorded using a thermal camera (TIM 450, Micro-Epsilon).

\section{Material behaviour and mesostructures}

Typical DSC curves of the various 3D printed PA6 films for the (a) 'in-plane' deposition mode, (b) ‘in-thickness’ deposition mode and (c) 'in-plane’ deposition mode with further CM are shown in Figure 4, 
with the melting point and crystallinity indicated assuming the equilibrium enthalpy of PA6 as $230 \mathrm{~J} / \mathrm{g}$ [14]. There are no significant differences in the melting temperatures of $\sim 200{ }^{\circ} \mathrm{C}$ for the various 3D printed PA6 films. Moreover, the degrees of crystallinity of the various 3D printed PA6 films are virtually identical, with values of $20.7 \%$, $21.0 \%$ and $20.4 \%$, respectively, being determined.

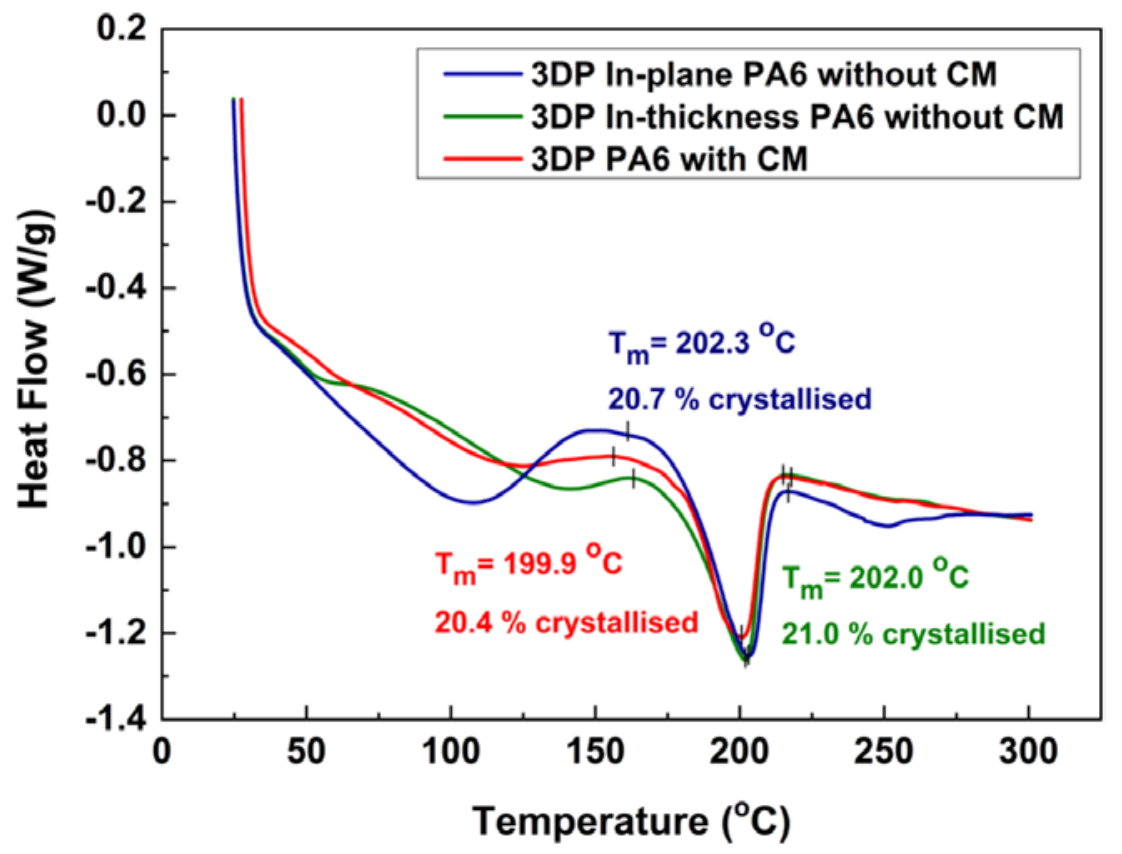

Figure 4. Typical DSC curves of the 3D printed PA6 films.

FTIR spectra of the 3D printed ‘in-plane’ deposition mode PA6 films, with and without further CM, are shown in Figure 5. As denoted in the spectra, characteristic bands, including the N-H stretching vibration (at $3290 \mathrm{~cm}^{-1}$ ), C-H stretching vibration (at $2925 \mathrm{~cm}^{-1}$ and $2850 \mathrm{~cm}^{-1}$ ), C=O stretching vibration (at $1637 \mathrm{~cm}^{-}$ ${ }^{1}$ ) and in-plane N-H deformation (at $1543 \mathrm{~cm}^{-1}$ ), can be seen clearly and these results are similar to the FTIR spectra results of PA6 reported by Ma et al. [15]. Hence, the chemical composition of the printed 'in-plane' deposition mode PA6 film with further CM was the same as that of the printed 'in-plane' deposition mode PA 6 film without further CM.

Therefore, the CM process did not alter the thermal or chemical properties of the printed PA6 polymer, which confirms the feasibility of testing the 3D printed 'in-plane' deposition mode films with further CM to obtain a benchmark test for assessing any increase in the extent and quality of the fusion bonding that may have resulted from the additional CM process. 


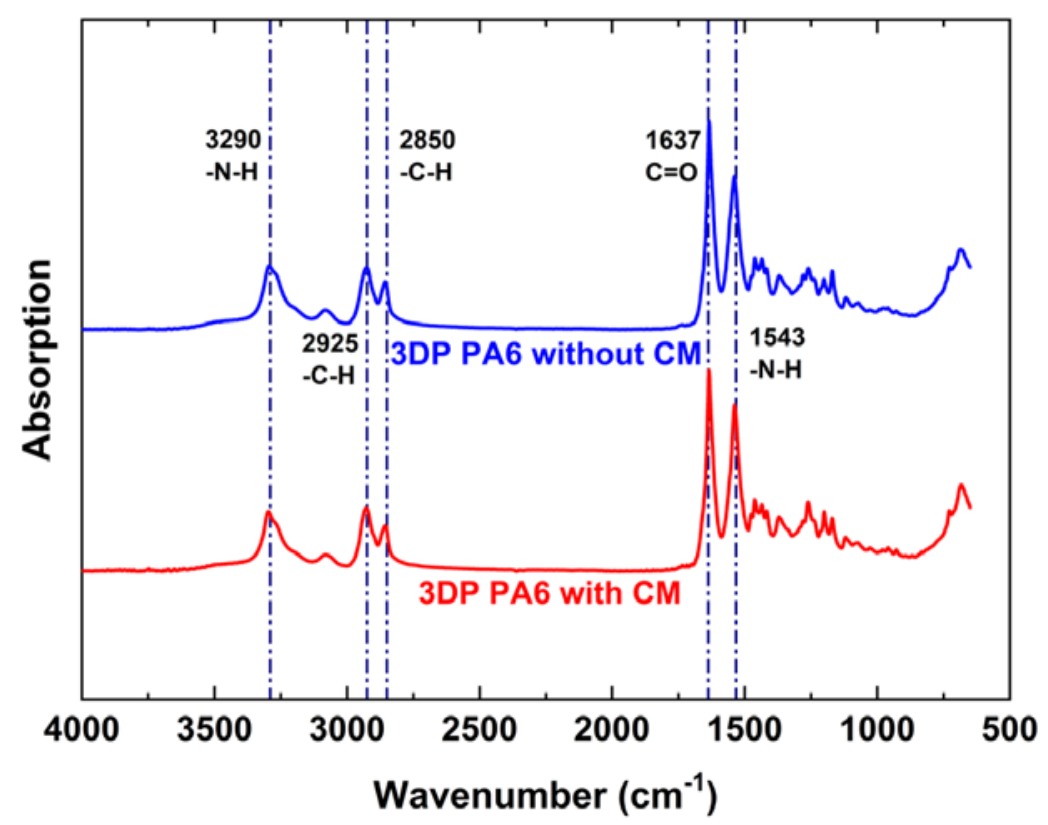

Figure 5. FTIR spectra of the 3D printed 'in-plane' deposition mode PA6 films, with and without further CM.

Typical cross-sections of the various thin films before EWF testing are shown in Figure 6. Local fluctuations in the thickness of the films can be seen clearly in the 3D printed PA6 films for both the 'in-plane' deposition mode and 'in-thickness' deposition mode films, which both possess a regular ridge-valley pattern. Specifically, the thickness ranges from 133 to $168 \mu \mathrm{m}$ (with an average of $142 \pm 14 \mu \mathrm{m}$ ) for the 'in-plane' deposition mode film, whereas the 'in-thickness' deposition mode film has a thickness which fluctuates between 311 to $401 \mu \mathrm{m}$ (with the average of $356 \pm 50 \mu \mathrm{m}$ ). Such variations in the thickness result from the transversely squeezed flow in the PA6 filaments under gravity and the depositing pressure from the nozzle. In comparison, the further CM process clearly helps to flatten the 'in-plane' deposition mode film (Figure 6(c)), showing a uniform thickness with an average value of $207 \pm 5 \mu \mathrm{m}$ for the film made using the 'in-plane' deposition mode with further CM processes. 


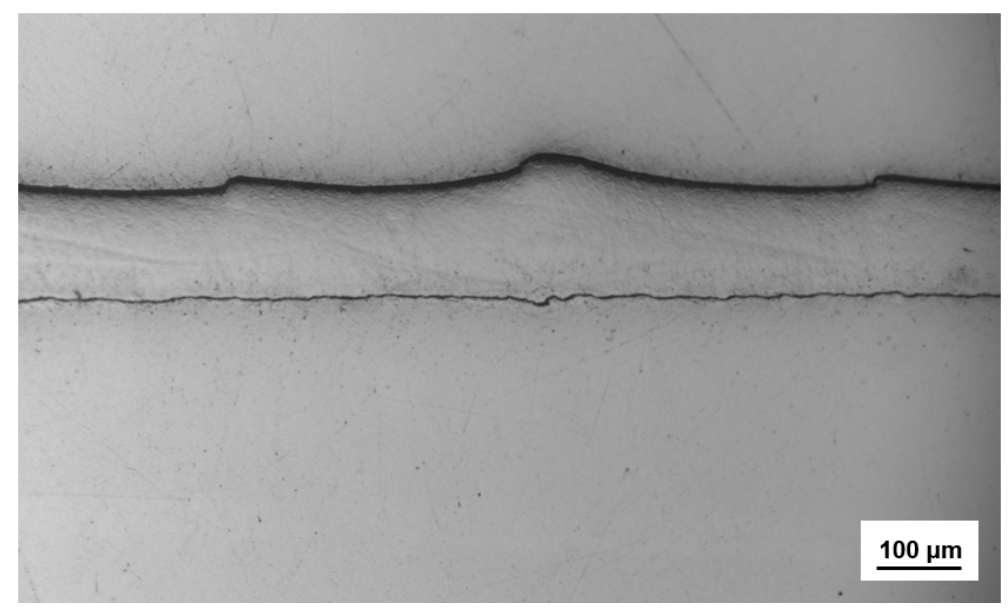

(a)

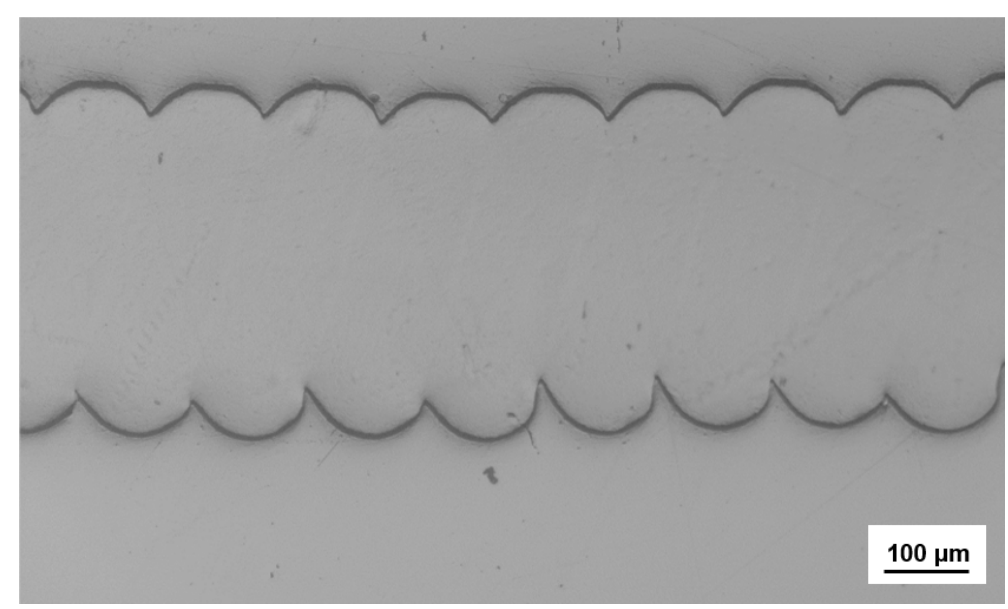

(b)

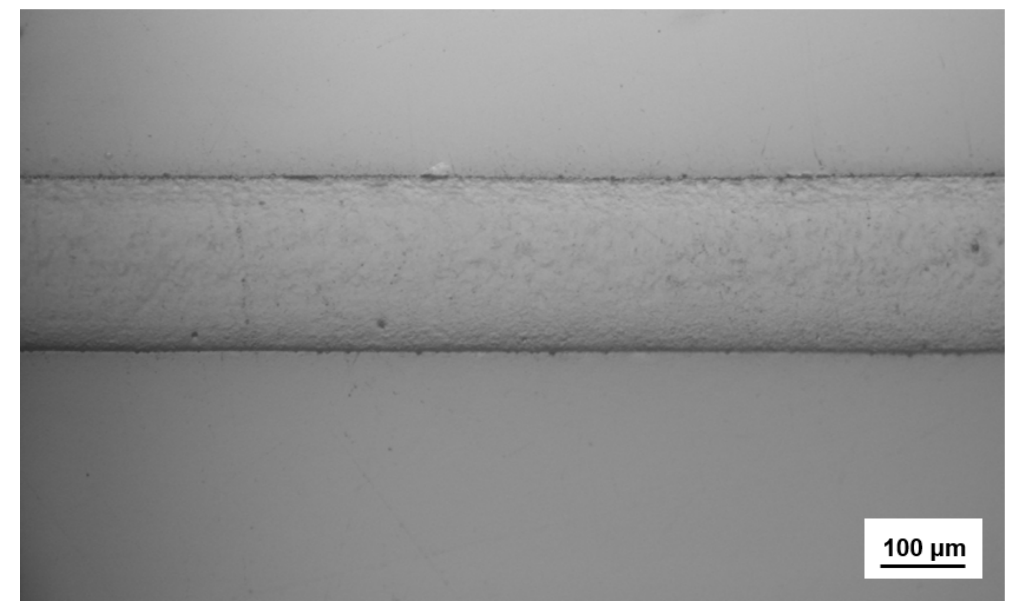

(c)

Figure 6. Optical micrographs of typical cross-sections of the various thin films for: (a) the 3D printed PA6 'in-plane' deposition mode, (b) the 3D printed PA6 'in-thickness' deposition mode and (c) the 3D printed PA6 ‘in-plane’ deposition mode with further CM. 


\section{Fusion bonding quality determined by the specific essential work of fracture}

\subsection{D printed single-layer PA6 films (i.e. the 'in-plane' deposition mode)}

Figure 7(a) shows typical load-displacement curves from the EWF tests with different ligament lengths for the 3D printed single-layer PA6 films (Figure 1(a)), to evaluate the fusion bonding between the PA6 filaments for this 'in-plane’ deposition mode. As is evident, all the load-displacement curves are highly similar, satisfying the critical prerequisite of the EWF method as discussed above [9]. It may also be observed that the area under the curves, after the maximum load has been attained, is much greater than that before the maximum load, indicating slow crack propagation, crack tip blunting and higher energy absorption, which is typical of the behaviour of ductile materials in EWF tests [16]. The fracture behaviour of an 'in-plane' deposition mode PA6 specimen with a nominal $10 \mathrm{~mm}$ ligament is shown in Figure 7(b), using screenshots taken at different stages from the video clip recorded during the test, namely, the start of the test, crack tip blunting, stable crack propagation and complete failure of the ligament. (The numbers on the load versus displacement curves in Figure 7(a) correspond to the numbers on the photographs shown in Figure 7(b).) With the increase in the applied tensile load, the sharp crack tips begin to blunt slightly, followed by the initiation of stable crack propagation immediately after the ligament fully yields. Also, a plastic zone with curved edges can be observed clearly around the growing crack tips, as there is a difference in colour due to the large deformation, i.e. thinning, of the film within the diffuse plastic region. In a valid EWF test, where the inherent material toughness can be assessed, complete ligament yielding does indeed precede the onset of crack initiation. That is, there should be a drop in the load in the corresponding load-displacement curve [17]. In the present work, analysis of the recorded video clips confirmed that cracks on both sides in all the EWF specimens began to propagate immediately after the load reached its maximum, which again indicates the validity of the current test results. Visual inspection of the specimen after EWF testing indicated that any elastic retraction of the fractured ligament was relatively very minor after the EWF test, revealing that the elastically stored energy in the specimen was released during the fracture process [9], as again required for a valid EWF test result. These observations were then confirmed by direct measurements, using digitised microscopy, on the test specimens. Typically, for example, the diffuse zone of the specimen having a ligament length of $10 \mathrm{~mm}$ was inspected and measured carefully just prior to fracture and after EWF testing. There was a reduction of less than $1 \%$ in 
the width and $15 \%$ in the height of the diffuse zone. This confirmed that the elastic retraction of the fractured ligament was indeed relatively minor after the EWF test had been completed and highlighting the full plasticity that developed within the diffuse zone.

The specific work of fracture, $w_{f}$, against ligament length, $L$, for the single-layer PA6 films is plotted in Figure 7(c), with the linear regression of the data as well as the 95\% confidence and prediction bands included. From a statistical analysis, the value of $w_{e}$, i.e. the positive intercept of the fitted regression line, was evaluated as being $25.7 \mathrm{~kJ} / \mathrm{m}^{2}$. Furthermore, the fit line demonstrates a very good linearity with a low degree of scatter, as manifested by the relatively high value of the coefficient of determination, $\mathrm{R}^{2}$, of 0.92 .

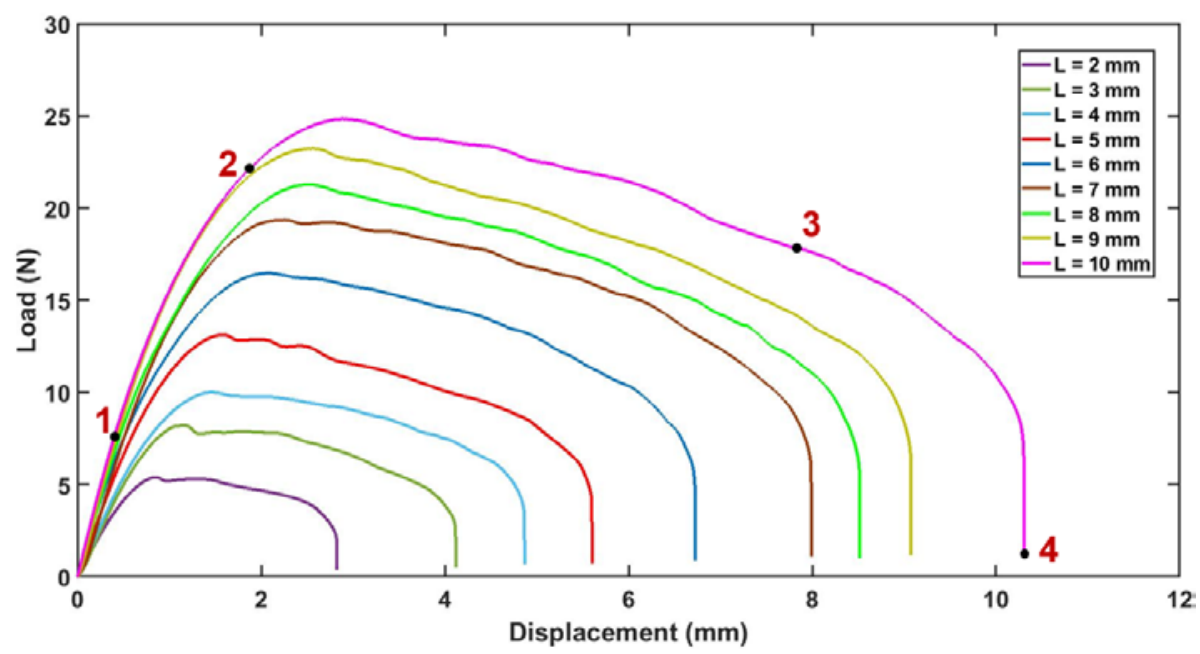

(a)

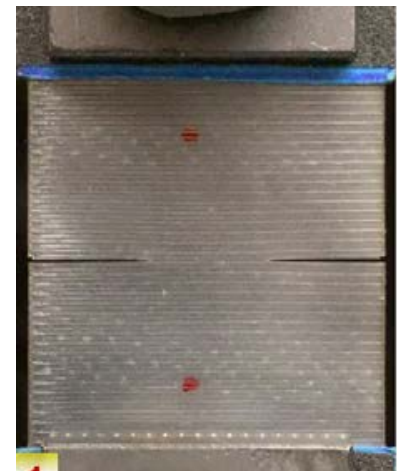

1

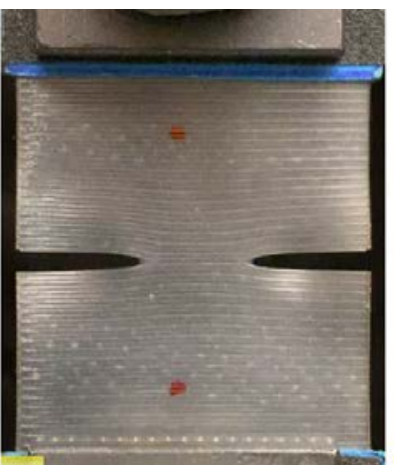

2

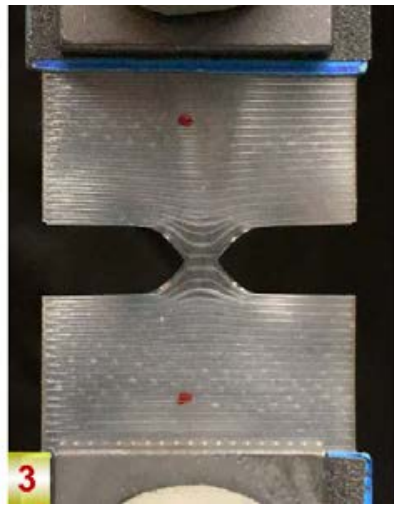

(b)

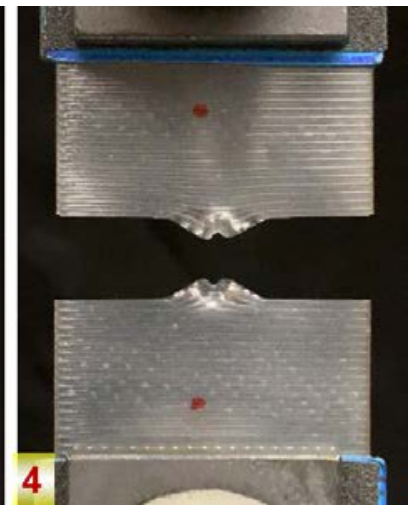

4 


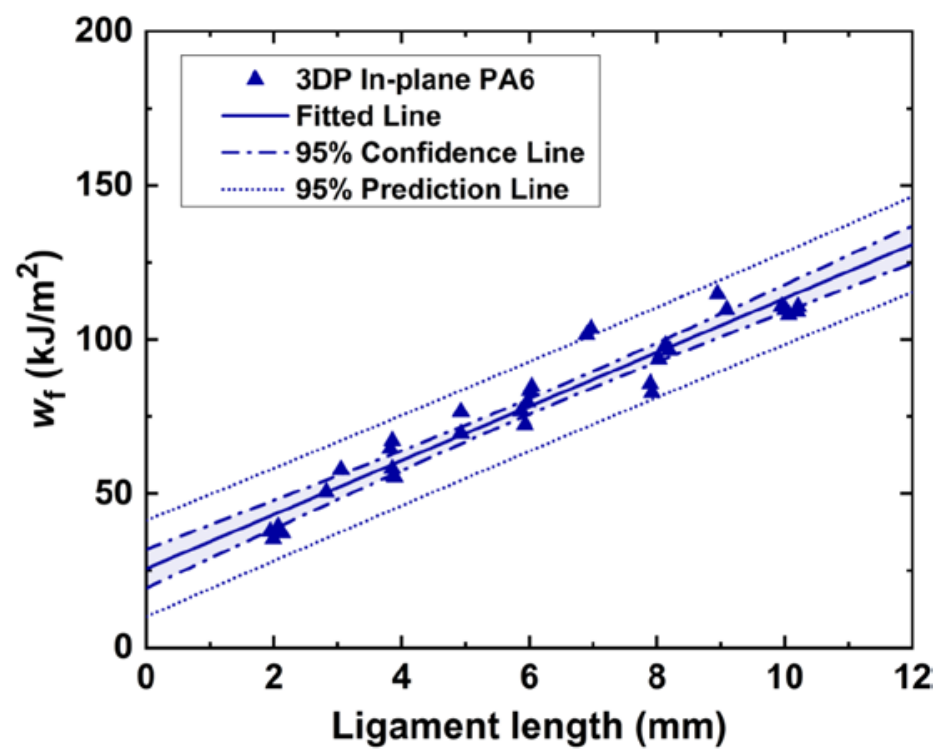

(c)

Figure 7. Results of the 3D printed, single-layer, ‘in-plane’ deposition mode PA6 films: (a) typical loaddisplacement curves, (b) fracture behaviour recorded during the test and (c) plot of the total specific work of fracture, $w_{f}$, against ligament length, $L$, with the $95 \%$ confidence and prediction lines shown. (The numbers on the load versus displacement curves in Figure 7(a) correspond to the numbers on the photographs shown

$$
\text { in Figure 7(b).) }
$$

\subsection{D printed single-filament PA6 films (i.e. the 'in-thickness’ deposition mode)}

EWF tests were also conducted on the 3D printed single-filament PA6 specimens along the z-axis (Figure 1(b)) to evaluate the fusion bonding between the filaments for the 'in-thickness' deposition mode, and to quantify the potential anisotropy developed during the FFF process. As shown in Figure 8(a), the representative load-displacement curves of the 3D printed PA6 films for the 'in-thickness' deposition mode generally demonstrate consistent trends, with the peak load being much greater than that found in the corresponding single-layer, 'in-plane’ deposition mode EWF test specimen for the same ligament length (Figure 7(a)). This much higher load arises from the greater thickness of the 'in-thickness' deposition mode EWF specimens of $356 \pm 50 \mu \mathrm{m}$, compared with $142 \pm 14 \mu \mathrm{m}$ for the 'in-plane' deposition mode specimens. In contrast, no apparent decrease is observed in the maximum displacements when final fracture occurs in these two types of 3D printed PA6 EWF test specimens. Moreover, the fracture behaviour of both the 'inplane' and the 'in-thickness' deposition mode EWF specimens is very similar, as was evident in the recorded 
videos, in which steady crack propagation and a distinct plastic zone is readily observed due to the thinning of the materials within a diffuse region during the EWF test (cf. Figures 7(b) and 8(b)).

Figure 8(c) shows the plot of the total specific work of fracture, $w_{f}$, against the ligament length, $L$, obtained from testing the 'in-thickness’ deposition mode PA6 films with different ligament lengths. Significant scatter is evident, with the data points spread broadly along the fitted line, yielding a low $\mathrm{R}^{2}$ at 0.50 and the value of $w_{e}$ is determined to be $21.3 \mathrm{~kJ} / \mathrm{m}^{2}$. Compared with the 'in-plane' deposition mode, the fusion bonding between filaments for the 'in-thickness' deposition mode specimens appears to be significantly poorer in both extent and quality, with a $17 \%$ decrease in $w_{e}$ and much larger confidence intervals. It would therefore appear that the value of $w_{e}$ and the coefficient of determination, $\mathrm{R}^{2}$, can be used as important indicators to quantify the extent and quality of the 3D printing using the FFF process, where for the latter aspect a higher value of $\mathrm{R}^{2}$ indicates a more efficient and robust printing configuration.

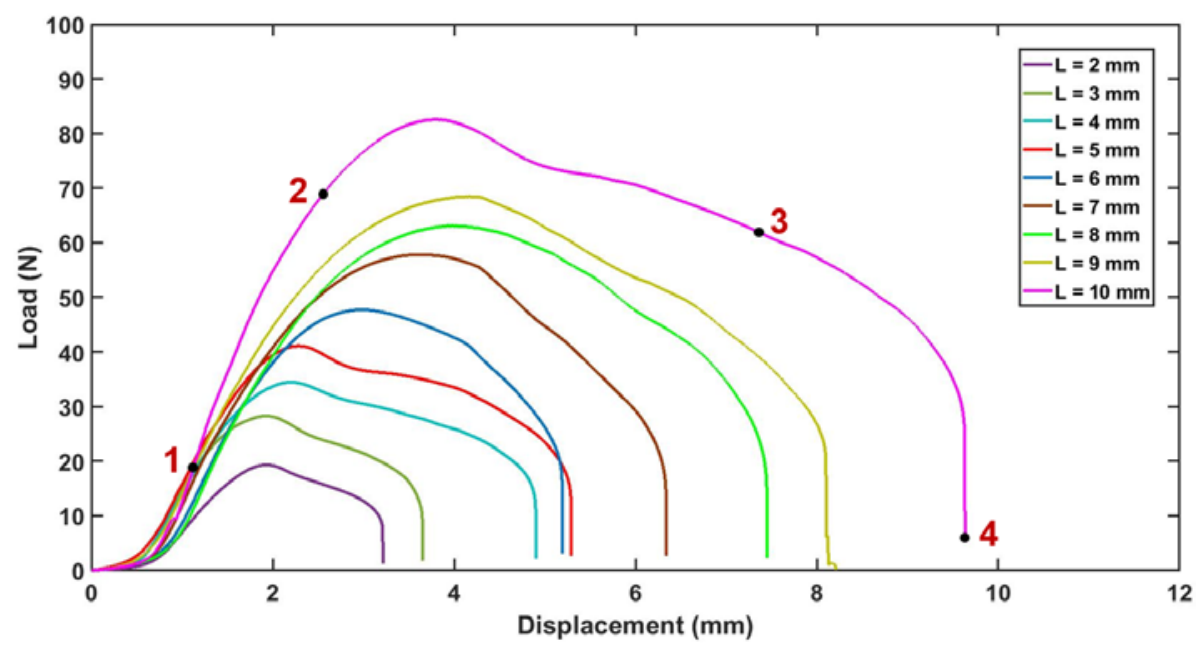

(a)

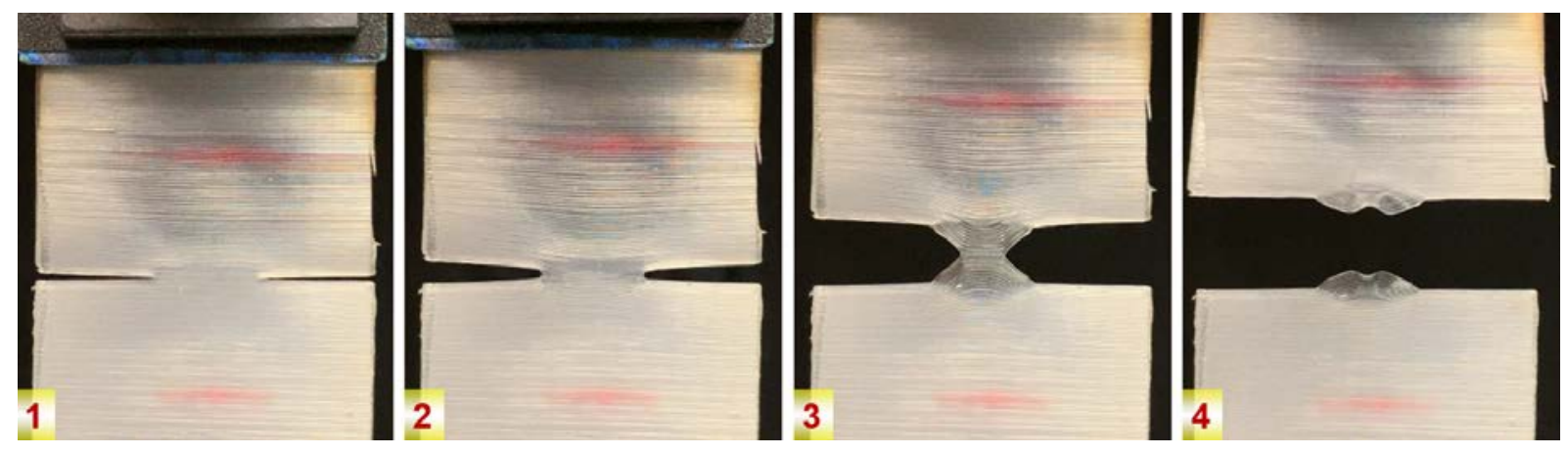

(b) 


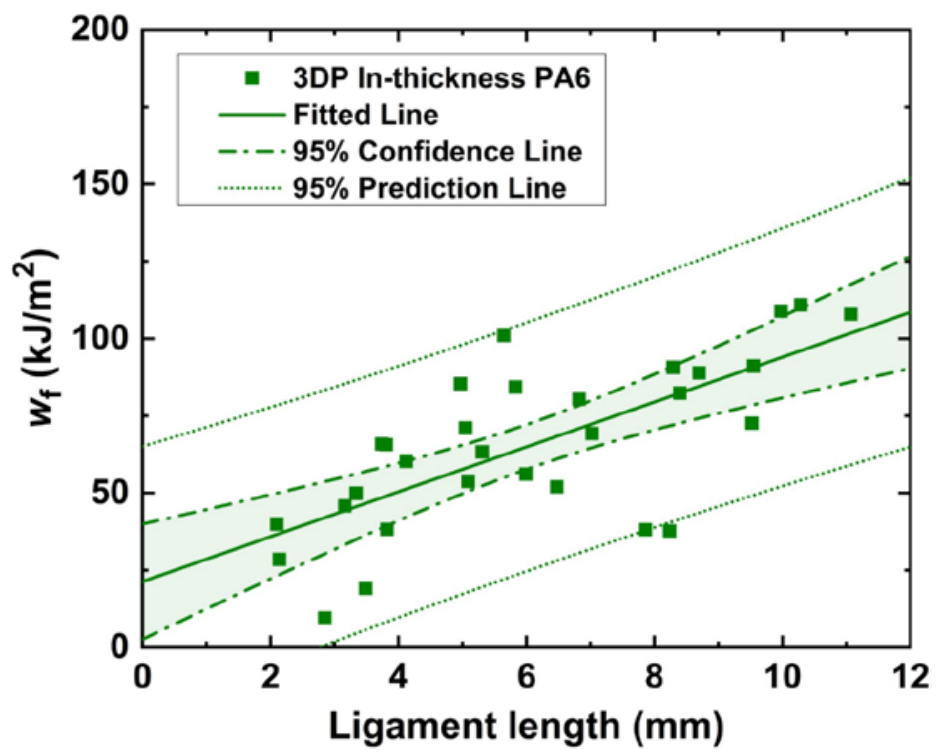

(c)

Figure 8. Results of the 3D printed, single-filament, 'in-thickness' deposition mode PA6 films: (a) typical load-displacement curves, (b) fracture behaviour recorded during the test and (c) plot of the total specific work of fracture, $w_{f}$, against ligament length, $L$, with the $95 \%$ confidence and prediction lines shown. (The numbers on the load versus displacement curves in Figure 8(a) correspond to the numbers on the photographs shown in Figure 8(b).)

\subsection{D printed ‘in-plane’ deposition mode PA6 films with further CM (i.e. the benchmark material)}

To provide a benchmark material, thin PA6 films were first 3D printed in the 'in-plane' deposited mode, and hot-pressed using CM to ensure thorough and uniform molecular inter-diffusion after the CM process. EWF tests were performed on the films made by such 3D printing in the 'in-plane' deposition mode plus further CM, and the results are shown in Figure 9. All the load-displacement curves in Figure 9(a) demonstrate a relatively high degree of consistency and, indeed, are highly similar in shape. In comparison with the results observed from by testing the 'in-plane' deposition mode PA6 films without CM, cf. Figure 7(a), there is a slight decrease in the maximum displacement, attributed to a lack of thinning of the polymer because the ridge-valley pattern was flattened during the CM process, see Figure 6. The difference in appearance of the EWF specimens resulting from this mesostructural variation (see Figure 6) is shown more clearly in Figure 9 (b), where a uniform white, smooth surface is observed. In addition, the typical fracture behaviour, including crack tip blunting in the DENT specimen and the steady propagation of sharp crack tips, can be seen. From 
plotting the value of $w_{\mathrm{f}}$ against $L$ (Figure 9 (c)), the value of $w_{e}$ was determined to be $38.1 \mathrm{~kJ} / \mathrm{m}^{2}$, which agrees well with the findings of previous studies in $[16,18]$, i.e. $32.5 \mathrm{~kJ} / \mathrm{m}^{2}$ and $36.9 \mathrm{~kJ} / \mathrm{m}^{2}$, respectively, for a PA6 polymer. Furthermore, the fitted line demonstrates a very low degree of associated scatter, as is indicated by the high value of the coefficient of determination, $\mathrm{R}^{2}$, of 0.96 and the very narrow $95 \%$ confidence and prediction lines.

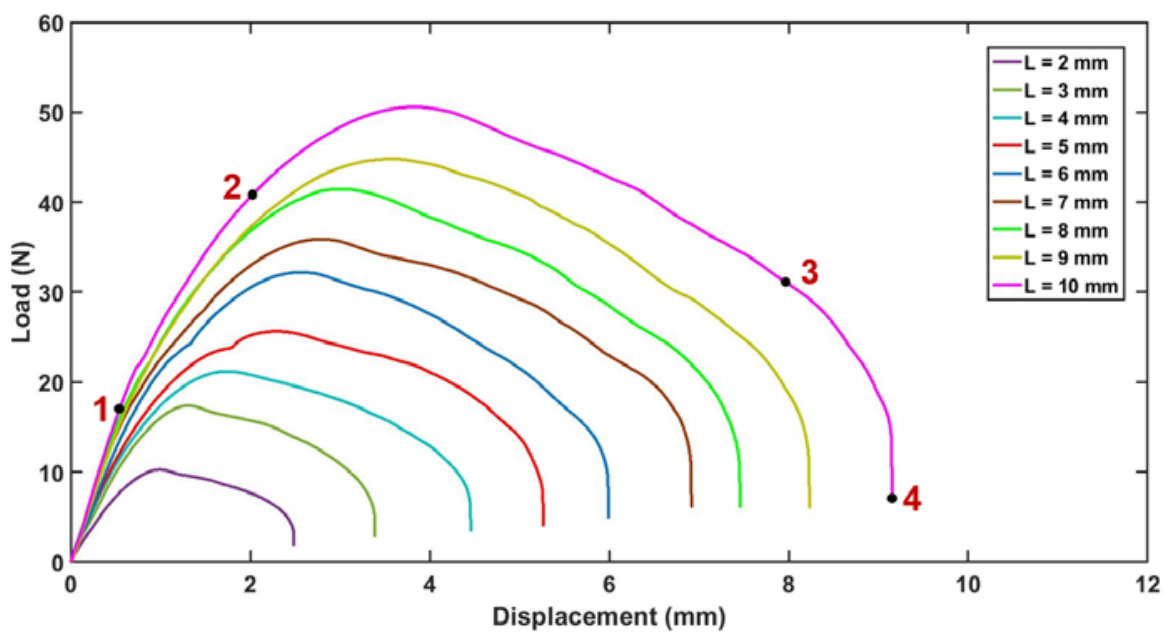

(a)
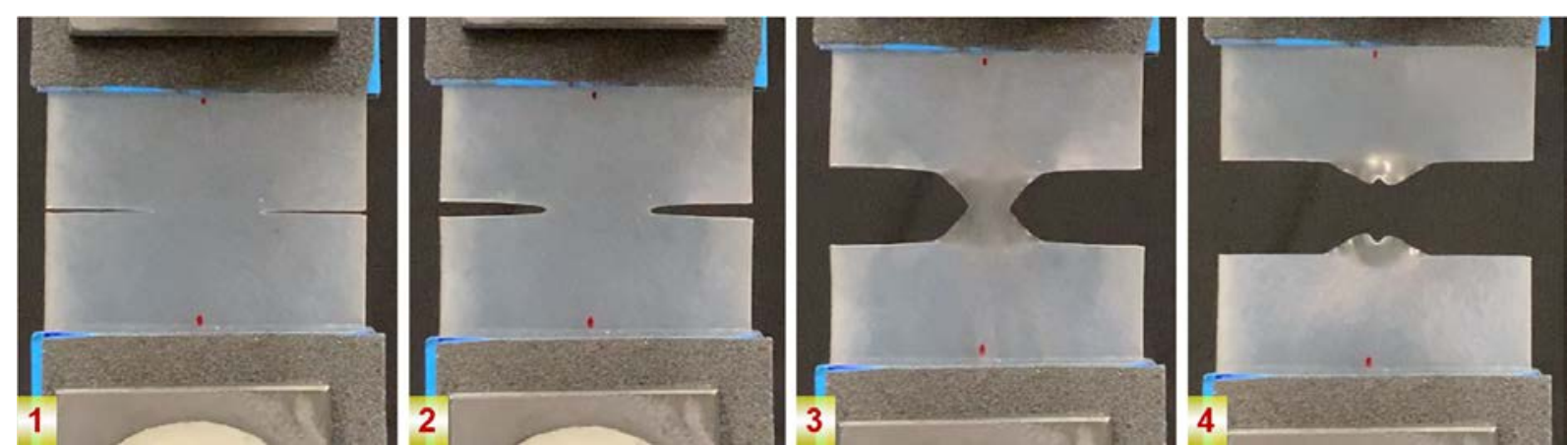

(b)

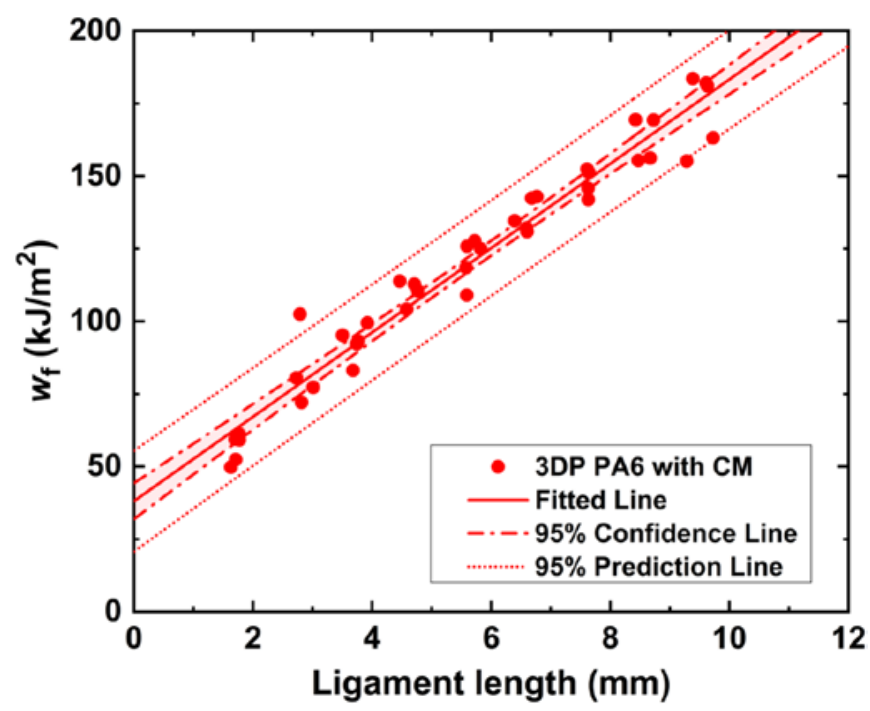

(c) 
Figure 9. Results of the 3D printed, ‘in-plane’ deposition mode with further CM PA6 films: (a) typical loaddisplacement curves, (b) fracture behaviour recorded during the test and (c) plot of the total specific work of fracture, $w_{f}$, against ligament length, $L$, with the $95 \%$ confidence and prediction lines shown. (The numbers on the load versus displacement curves in Figure 9(a) correspond to the numbers on the photographs shown

$$
\text { in Figure 9(b).) }
$$

The results for the specific essential work of fracture, $w_{\mathrm{e}}$, and the coefficient of determination, $\mathrm{R}^{2}$, are summarised in Table 1, from which differences in these values for the various types of 3D printed PA6 films can be clearly seen. After further CM, the value of $w_{\mathrm{e}}$ for the benchmark material shows a significant increase (i.e. of $\sim 50 \%$ ) compared with that of the 3D printed, 'in-plane' deposition mode film, i.e. a film with no further CM. The enhancement for the value of $w_{\mathrm{e}}$ for the benchmark material is even more considerable when compared with the 3D printed, 'in-thickness' deposition mode film (i.e. of $\sim 80 \%$ ). Thus, these results demonstrate a very significant difference between the extent of fusion bonding in the various types of 3D printed polymer films and the major effect for the benchmark material which is achieved by a traditional (CM) manufacturing process being used after the FFF process. A factor that contributes to the sharp rise in the fracture toughness in the 3D printed, ‘in-plane’ deposition mode films with further CM can also be observed from examining the fracture surfaces using SEM. As shown in Figure 10(a) and (b), both the typical fracture surfaces of the 3D printed 'in-plane' and 'in-thickness' deposition mode EWF specimens look rather smooth, with no visible streaks left, even after relatively large deformations have taken place. In contrast, the fracture surface morphology after the EWF test (Figure 10(c)) is very different for the 3D printed, 'in-plane' deposition mode PA6 film fabricated by the FFF process plus using further CM, where a much rougher surface with more severe plastic deformation can be readily seen. This clearly demonstrates that more energy is indeed absorbed during the fracture test, resulting in the higher value of $w_{\mathrm{e}}$ that is measured for the benchmark material. 
Table 1. The EWF results for the various types of 3D printed PA6 films

\begin{tabular}{cccc}
\hline & 'In-plane' & 'In-thickness’ & $\begin{array}{c}\text { 'In-plane’ with further CM } \\
\text { (i.e. the benchmark material) }\end{array}$ \\
\hline$w_{\mathrm{e}}\left(\mathrm{kJ} / \mathrm{m}^{2}\right)$ & $25.7 \pm 3.1^{*}$ & $21.3 \pm 9.1^{*}$ & $38.1 \pm 3.1^{*}$ \\
$\mathrm{R}^{2}$ & 0.92 & 0.50 & 0.96 \\
\hline
\end{tabular}

${ }^{*}$ The 95\% confidence interval is shown.

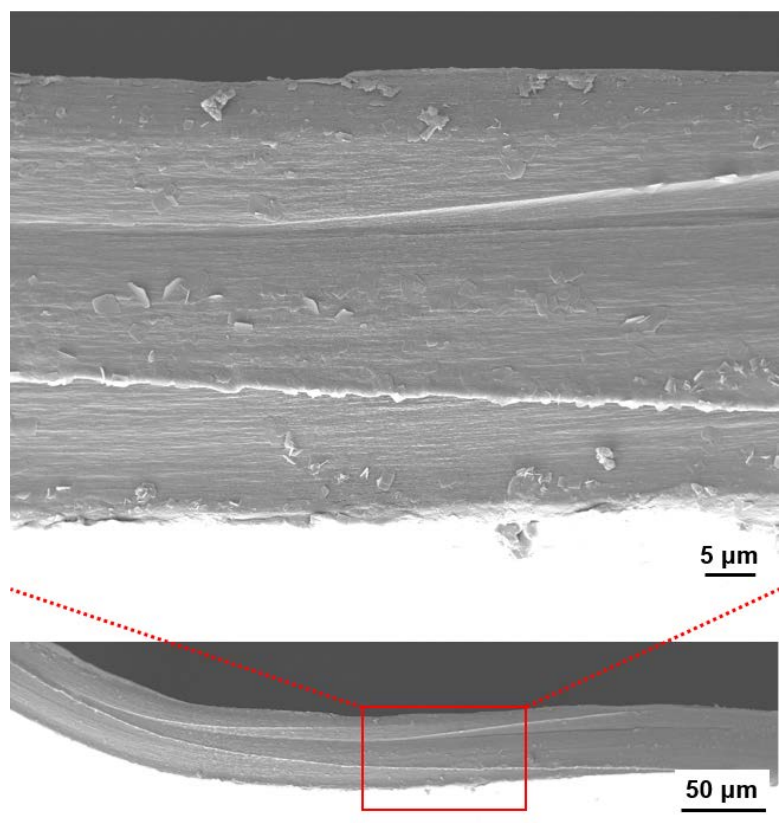

(a)

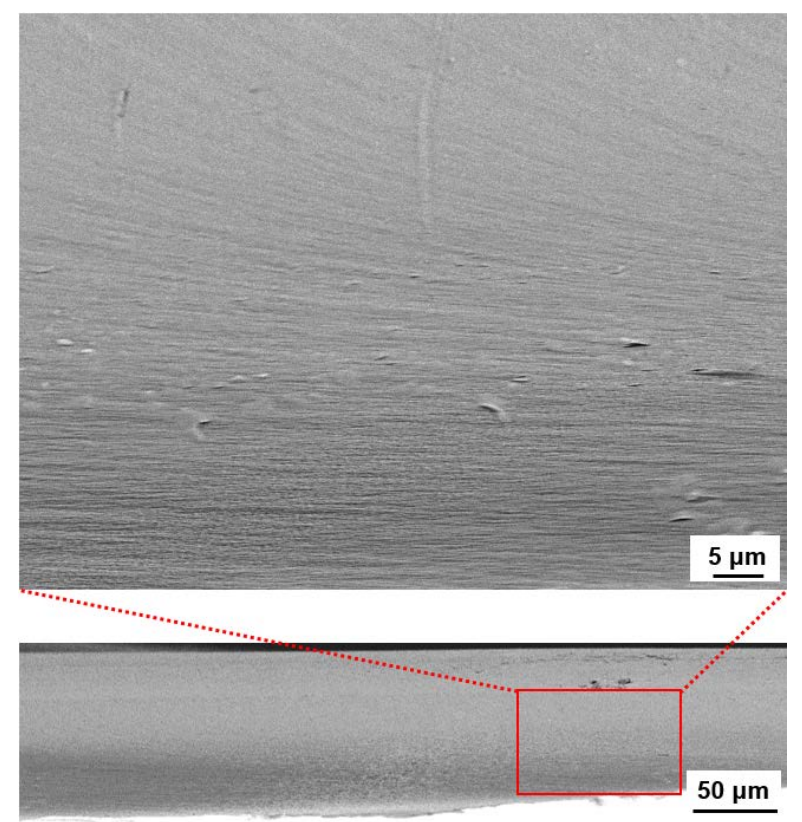

(b)

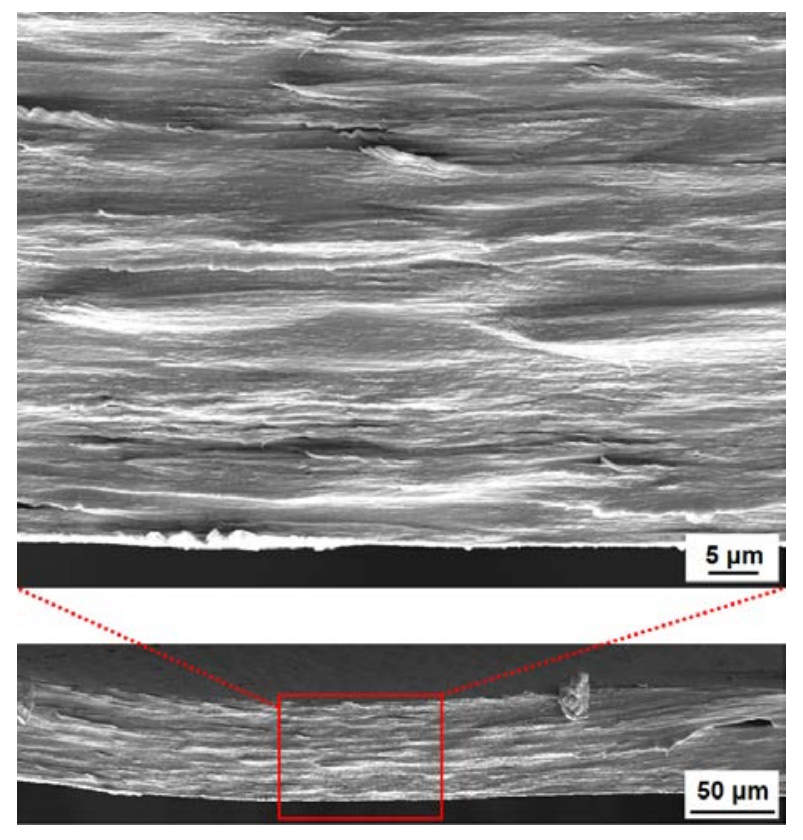

(c)

Figure 10. SEM images showing typical fracture surfaces of the 3D printed PA6 films after EWF testing: (a) the 'in-plane' deposition mode film, (b) the 'in-thickness' deposition mode film and (c) the 'in-plane' deposition mode film with further CM (i.e. the benchmark material). 
Considering the mechanisms for the above observations, during the FFF process a molten PA6 filament is deposited next to ('in-plane'), or above ('in-thickness'), a cooled PA6 filament deposited previously. The molten filament first forms intimate contact with the cold filament. Heat transfer causes the previous filament to melt, promoting molecular inter-diffusion across the interface between the two filaments. The quality and extent of the fusion bonding is dependent mainly on the degree of molecular inter-diffusion and, therefore, thorough and uniform molecular inter-diffusion leads to a higher value of $w_{\mathrm{e}}$, as demonstrated by the PA6 film fabricated by the FFF process plus further CM. Now, the value of $w_{\mathrm{e}}$ can also be affected by the crystallisation behaviour of PA6 during the thermal cycle incurred. However, the results in Figure 4 confirmed that the degrees of crystallinity in the 3D printed 'in-plane' deposition mode PA6 films, without or with further CM, are almost identical.

Finally, considering the values of the coefficient of determination, $\mathrm{R}^{2}$, calculated from the EWF test data, Table 1 reveals noticeable differences for the various types of 3D printed PA6 films. As may be seen, the value of $\mathrm{R}^{2}$ is clearly highest for the benchmark material, i.e. when further CM is employed following the FFF process. Indeed, it is evident that a smaller value of $\mathrm{R}^{2}$ is indicative of a decline in the quality, i.e. consistency, of the fusion bonding, which is of major importance when a consistent fabrication quality is required in practical applications.

\section{Further discussions}

\subsection{The scatter in the results for the 3D printed single-filament, 'in-thickness' deposition mode PA6 film}

As shown in Table 1, the value of $\mathrm{R}^{2}$ of the fitted line from the EWF tests for the 3D printed singlefilament, 'in-thickness’ deposition mode PA6 films is significantly lower than the value of $\mathrm{R}^{2}$ for the singlelayer, 'in-plane' deposition mode PA6 films. The factors that lead to the lower average value of $w_{\mathrm{e}}$ and the significantly greater scatter for the 'in-thickness' deposition mode films are insufficient and poor reproducibly of the fusion bonding between the filaments in these films. As may be seen in Figure 11, a bondline can be clearly observed between every two adjacent filaments in the 3D printed single-filament film, i.e. for the 'inthickness' deposition mode film. The possible cause of such a mesostructural pattern is the different cooling 
rates within individual filaments after deposition, because the temperature of the shell, i.e. the boundary on the filament, decreases more rapidly due to a much larger temperature gradient, as seen in the modelling studies by Gantenbein et al. [19].

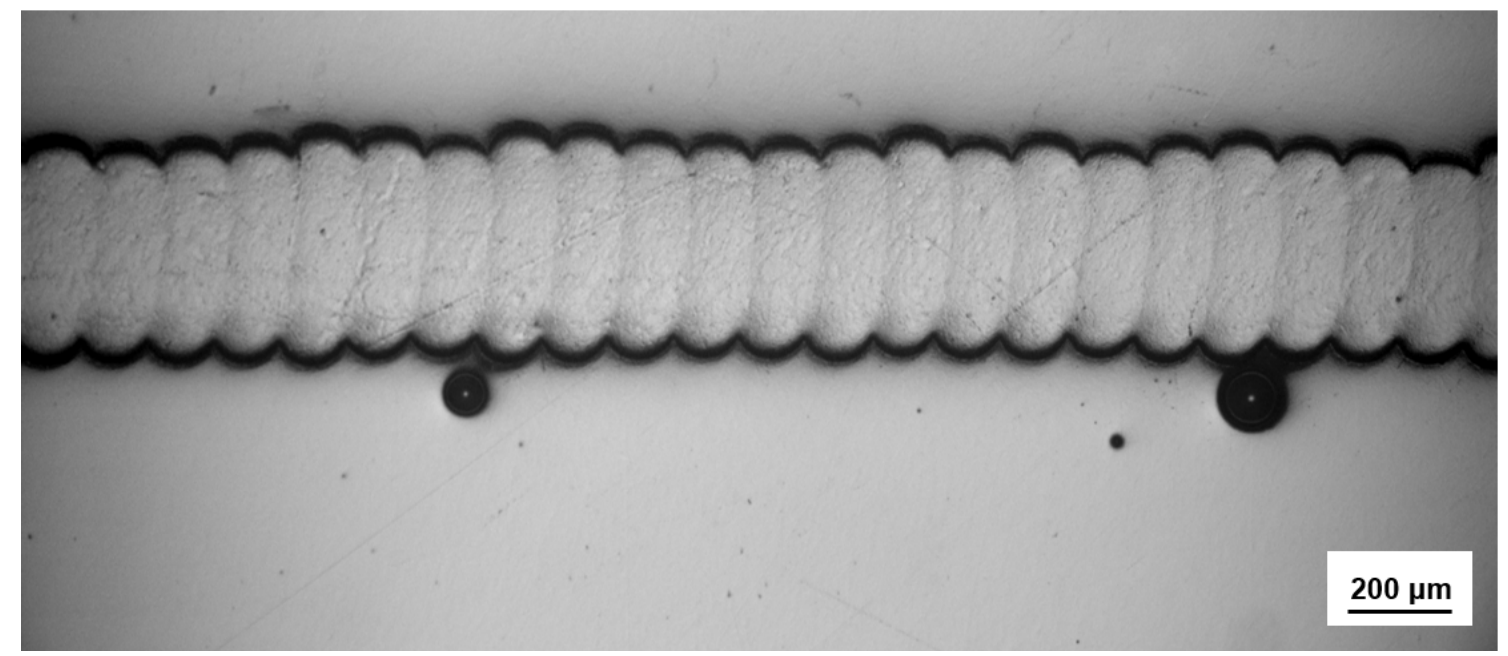

Figure 11. Optical micrograph of a 3D printed single-filament ('in thickness’ deposition mode) film showing the bondline.

\subsection{Effects of variations in the cross-sectional thickness}

A post-mortem on the fracture surfaces was also performed from a side-view to gain a better understanding of the fracture mechanisms in the PA6 films after EWF testing and to study any variations in the cross-sectional thickness of the 3D printed films (cf. Figure 6). The side-view of the diffuse zone in specimens after EWF testing, from the 'in-plane' and 'in-thickness' deposition mode films, as well as the 'inplane' deposition mode film with further CM process, can be seen in Figure 12 with the fractured ligament positioned at the right-hand side. For the ‘in-plane’ film (see Figure 12(a)), compared with the regular ridgevalley pattern shown at the left-hand side, the thinning of the material within the diffuse zone is easily distinguished, as confirmed by about a 50\% reduction in the average thickness measured from the optical micrograph. Additionally, the difference in height between the ridge and valley is much less in the diffuse zone, i.e. decreasing from $29 \mu \mathrm{m}$ to $13 \mu \mathrm{m}$, due to the substantial extension and plastic flow of the diffuse zone during the EWF test. This confirms the fact that the variation in thickness of the ridge-valley pattern does not cause premature failure. Similarly, a significant amount of deformation is readily observed in the 3D printed 'in-thickness' deposition mode film in Figure 12(b), as indicated by the three dashed lines connecting 
the ridges of the corresponding individual deposited filaments. The significant relative displacement of the peaks and a reduction of about $45 \%$ in the thickness within the diffuse zone suggest that the material has been fully yielded, which confirms that the fracture mechanism is not affected by the stress concentrations on the wrinkled surface of the printed film. Such an argument can be further supported by the difference in the height between the peak and the valley, which drops significantly from $73 \mu \mathrm{m}$ to $13 \mu \mathrm{m}$. In comparison, the reduction in thickness in the diffuse zone for the film from 'in-plane' deposition with further CM is 57\%, i.e. from 184 $\mu \mathrm{m}$ to $78 \mu \mathrm{m}$ as marked in Figure 12(c). These detailed observations and measurements confirm the ductile fracture behaviour with a diffuse zone in the printed films during EWF testing.

For the printed films from the 'in-plane and the 'in-thickness' deposition, even though the variations in thickness within the diffuse zone were found to decrease significantly, there is still some minor fluctuation in the thickness of the film due to the nature of the FFF process which is likely to contribute to scatter in the test results, i.e. a lower value of $\mathrm{R}^{2}$. However, considering the relatively small standard deviation associated with the average thickness (see Figure 6), i.e. of $10 \%$ and 14\%, respectively, for the 'in-plane' deposition film and the 'in-thickness' deposition film, the quantitative test results obtained in the present study are considered to be sound and useful for comparative purposes. Therefore, the relatively large difference in the extent and quality of the fusion bonding between the 3D printed PA6 in both the 'in-plane' and 'in-thickness' deposition modes and the benchmark material, i.e. using the 'in-plane’ deposition mode with further CM, is indeed a very valid observation.

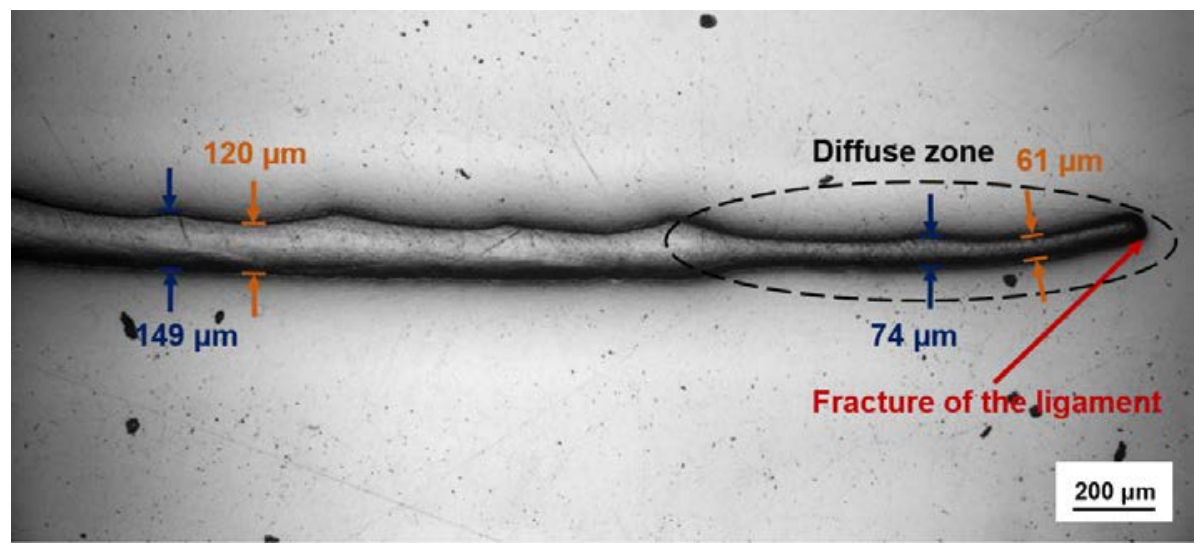

(a) 


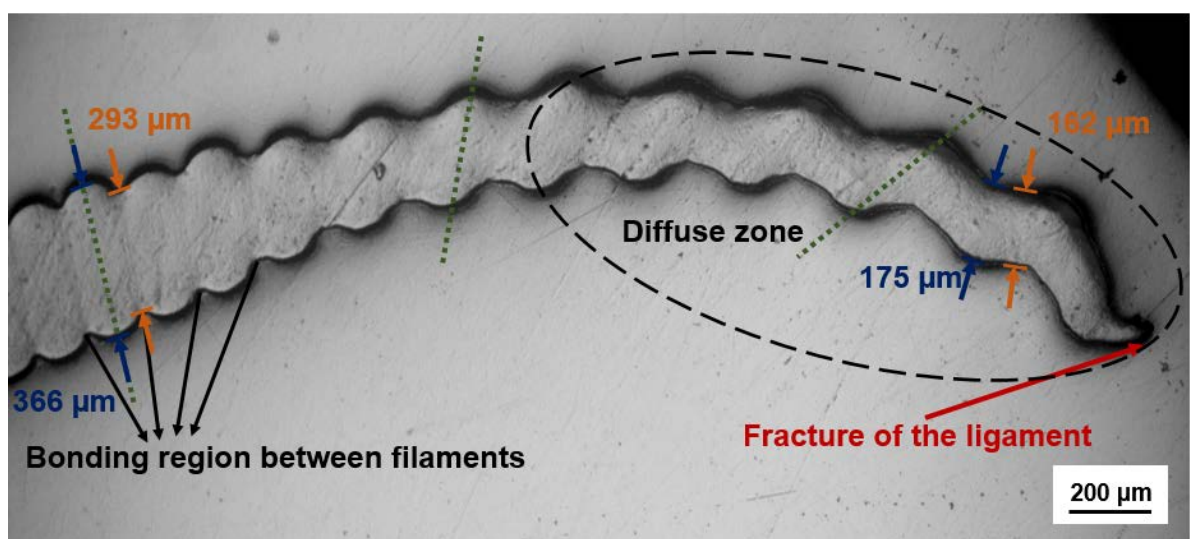

(b)

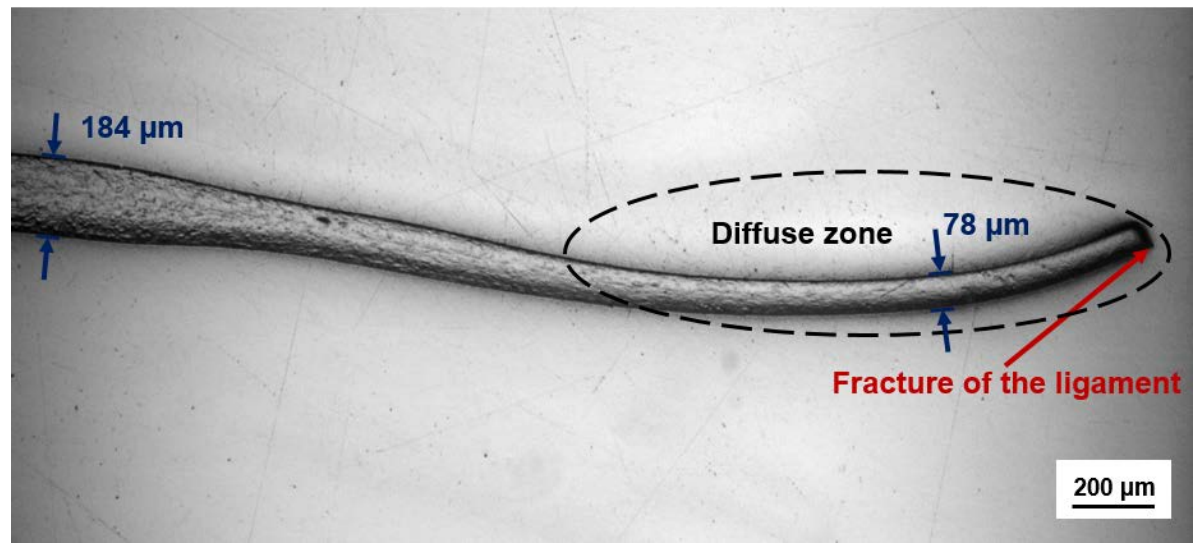

(c)

Figure 12. Side-view optical micrographs of the fracture surfaces of PA6 films after EWF testing with changes in thickness of the diffuse zone indicated: (a) 'in-plane' deposition mode film, (b) 'in-thickness' deposition mode film and (c) the ‘in-plane’ deposition mode with further CM film.

\subsection{Effects of the diffusion temperature on the fusion bonding}

In an FFF process, the temperature is known to be a critical parameter that influences the extent and quality of the fusion bonding, i.e. adhesion, between the deposited filaments. To investigate the relationship between the processing temperature and the fusion bonding attained, as characterised by the EWF tests, the thermal history of the materials was monitored using a thermal camera during the FFF process, with the emissivity of the camera set at 0.92 in accordance with the work of Vaes et al. [20]. The temperature distributions during the 3D printing of the single-layer, 'in-plane' deposition mode film (Figure 1(a)) and the single-filament 'in-thickness’ deposition film (Figure 1(b)) are shown in Figures 13(a) and (b), respectively. The temperature of the single-layer film is clearly much higher than that of the single-filament film, although the printing temperature, i.e. of $275^{\circ} \mathrm{C}$, was the same for both types of film. In the single-layer, 'in-plane' 
deposited mode PA6 film, the building plate and previously deposited fused filaments provide considerable thermal inertia, which helps significantly to reduce the heat loss via convection. In contrast, in the 3D printing of the single-filament 'in-thickness' deposited mode PA6 film, previously deposited filaments have already cooled to room temperature, suggesting that much less thermal energy is available to promote molecular interdiffusion, hence leading to a lower extent of fusion bonding and a weaker interface between the filaments.

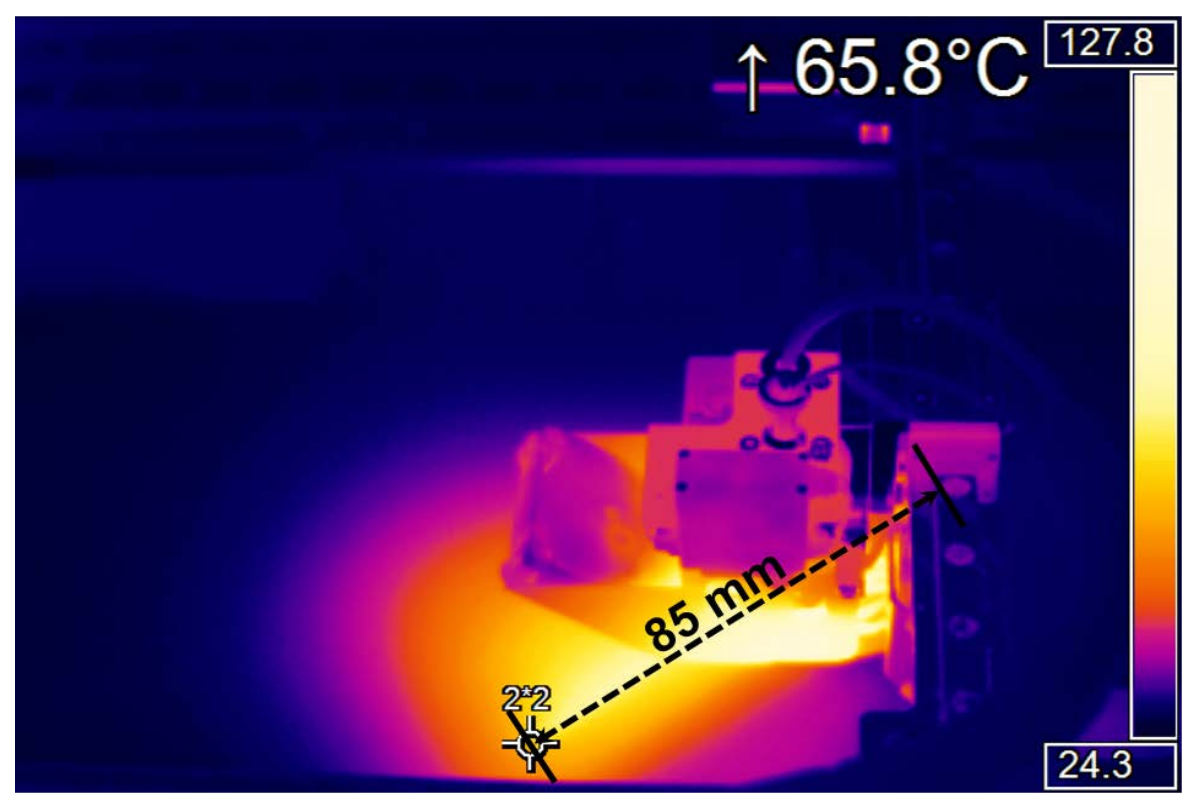

(a)

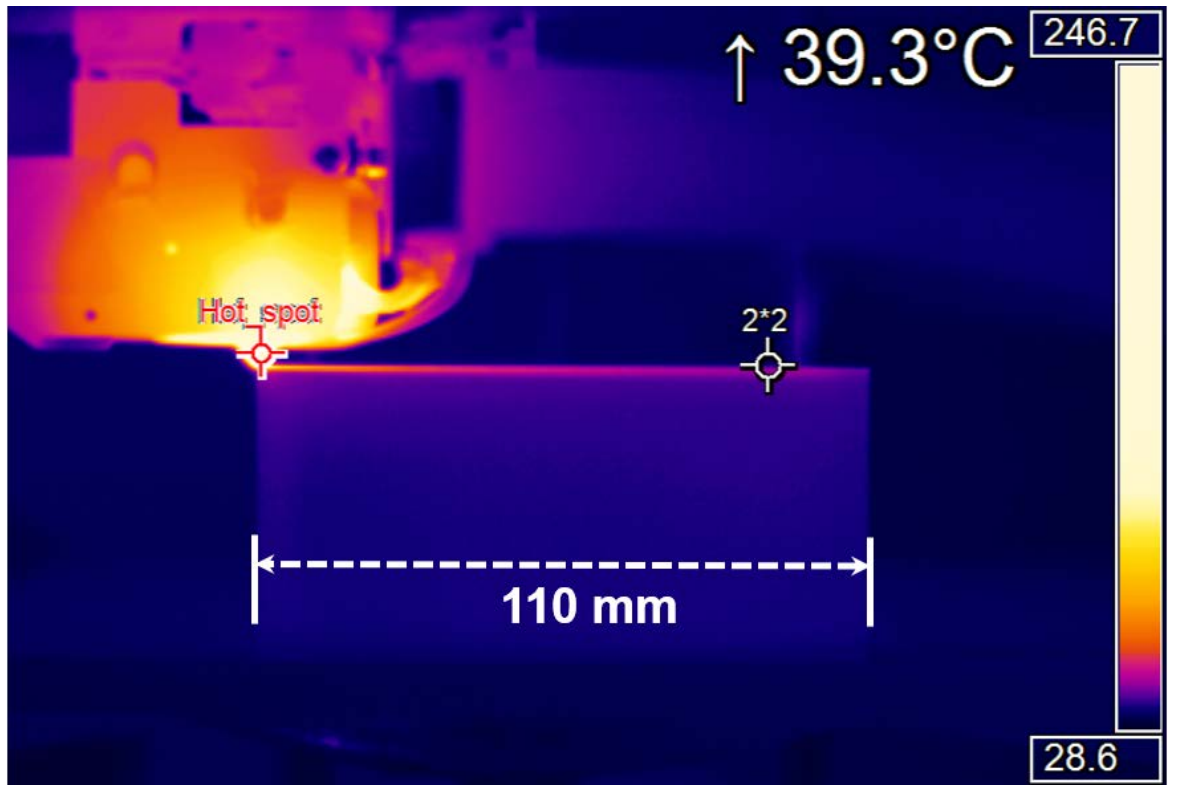

(b)

Figure 13. Thermal images showing the temperature distribution when $3 \mathrm{D}$ printing using the PA6 feedstock materials, with a $2 \times 2$ measuring area pinned at a location when the deposited materials had cooled for 5 
seconds: (a) single-layer, 'in-plane’ deposited mode film (top-view) and (b) single-filament, 'in-thickness' deposited mode (side-view).

A measurement area of $2 \times 2$ pixels was selected to post-process the recorded thermal videos to quantify the transient thermal history of an individual PA6 filament just after deposition, while 3D printing in the 'in-plane' and 'in-thickness' deposition modes, respectively, as indicated in Figure 13. As may be seen in Figure 14, it only takes about 1.65 seconds for the filament to cool from a peak temperature of $246.5{ }^{\circ} \mathrm{C}$ to 47 ${ }^{\circ} \mathrm{C}$, i.e. the glass transition temperature of PA6, when printing in the 'in-thickness' deposition mode. It is worth noting that only a portion of the thermal history can be captured when 3D printing in the 'in-plane' deposition mode (Figure 14), since the print-head blocks the view of the camera until the head moves clear. However, the temperature of the portion detected during printing of the single-layer film is clearly much higher than that observed in the single-filament film. For instance, the temperature is $66^{\circ} \mathrm{C}$ after cooling for $\sim 5$ seconds in the single-layer film, whereas it is $39{ }^{\circ} \mathrm{C}$ in the single-filament film. Considering the rapid cooling of the extruded filaments, and therefore the very short time available for inter-diffusion in the single-filament, 'in-thickness' deposition mode, the sharp fall in the temperature obviously results in insufficient molecular mobility for good and consistent fusion bonding, which in turn yields a somewhat lower average value of $w_{\mathrm{e}}$ and a significantly lower value of $\mathrm{R}^{2}$. 


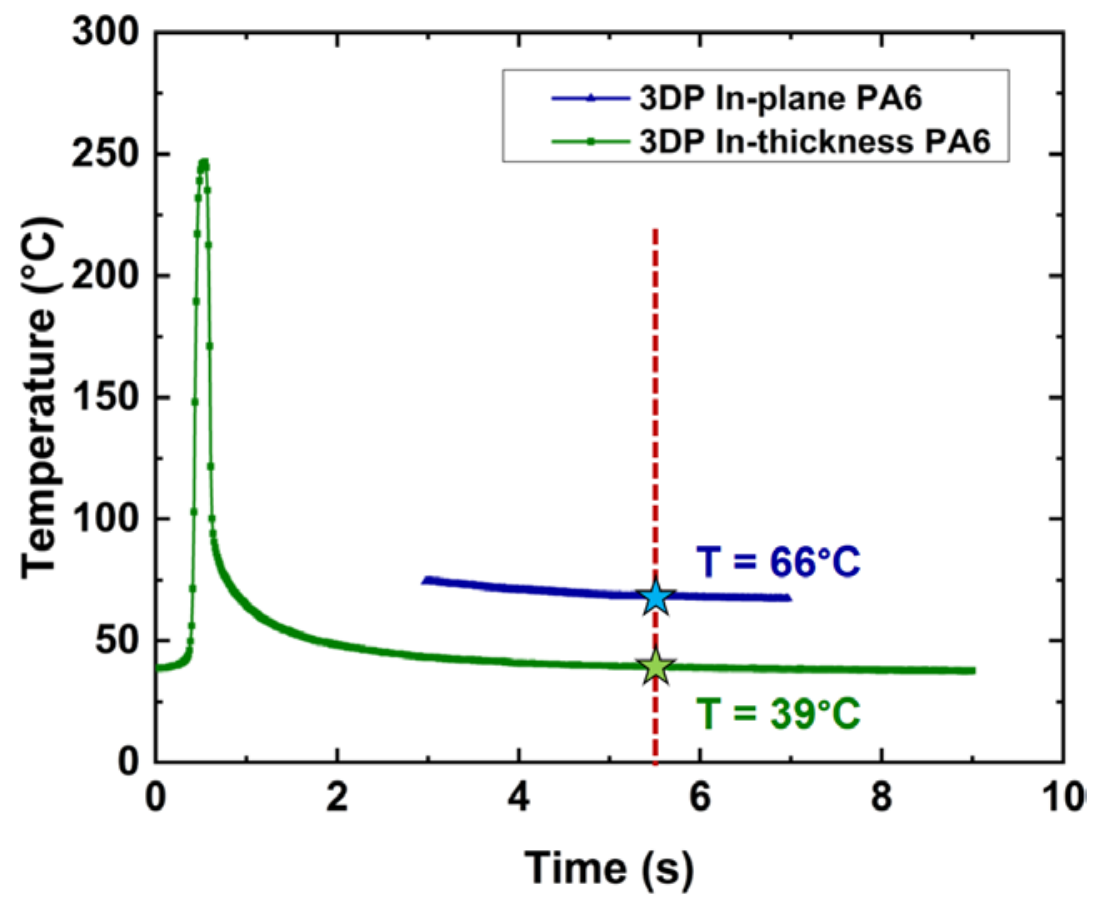

Figure 14. Thermal history of a single PA6 filament during the FFF process when 3D printing (3DP) in the single layer, 'in-plane’ deposition mode and in the single-filament, 'in-thickness’ deposition mode.

To further quantify the effects of temperature on the mechanical properties of the printed PA6 films, nanoindentation tests were conducted using a Hysitron nanoindentation testing system by a load control method using a conical indenter. The loading time, holding time and unloading time were all set to be 10 seconds, with a peak load of $1 \mathrm{mN}$. A printed single-layer film and a single-filament film of appropriate size were cast in the same mould using an epoxy-based embedding material, followed by careful polishing to obtain a flat cross-section. As indicated in the micrographs shown in Figure 15(a) and (b), twenty areas to undertake the nanoindentation tests, with a horizontal distance of about $40 \mu \mathrm{m}$ between adjacent points, were selected. Figure 15(c) shows a typical load-depth curve of the conical indentation test for a test area on 3D printed PA6 films for both the 'in-plane' and 'in-thickness' deposition modes. The modulus and hardness in each test were determined from the unloading portion of the corresponding load-depth curve, in accordance with Oliver and Pharr's method [21], and the results are shown in Figure 15(d). For the 3D printed 'in-plane' deposition mode, i.e. for the single-layer film, the results clearly reveal a greater stiffness and a smaller final indention depth, $h_{\mathrm{f}}$, compared with the single-filament, 'in-thickness' deposition mode film. Moreover, both the modulus and hardness are clearly higher for the 'in-plane' deposition mode film, with values of $0.39 \pm$ 
$0.01 \mathrm{GPa}$ and $28.9 \pm 0.7 \mathrm{MPa}$, respectively. These values may be compared with values of $0.36 \pm 0.01 \mathrm{GPa}$ and $26.7 \pm 0.5 \mathrm{MPa}$ for the 'in-thickness' deposition mode film. Therefore, the single-layer, 'in-plane' deposition mode film exhibits better mechanical properties which can be attributed to the development of better fusion bonding at the higher temperatures experienced during the FFF process, compared with the FFF process when 3D printing the single-filament, 'in-thickness’ deposition mode film.

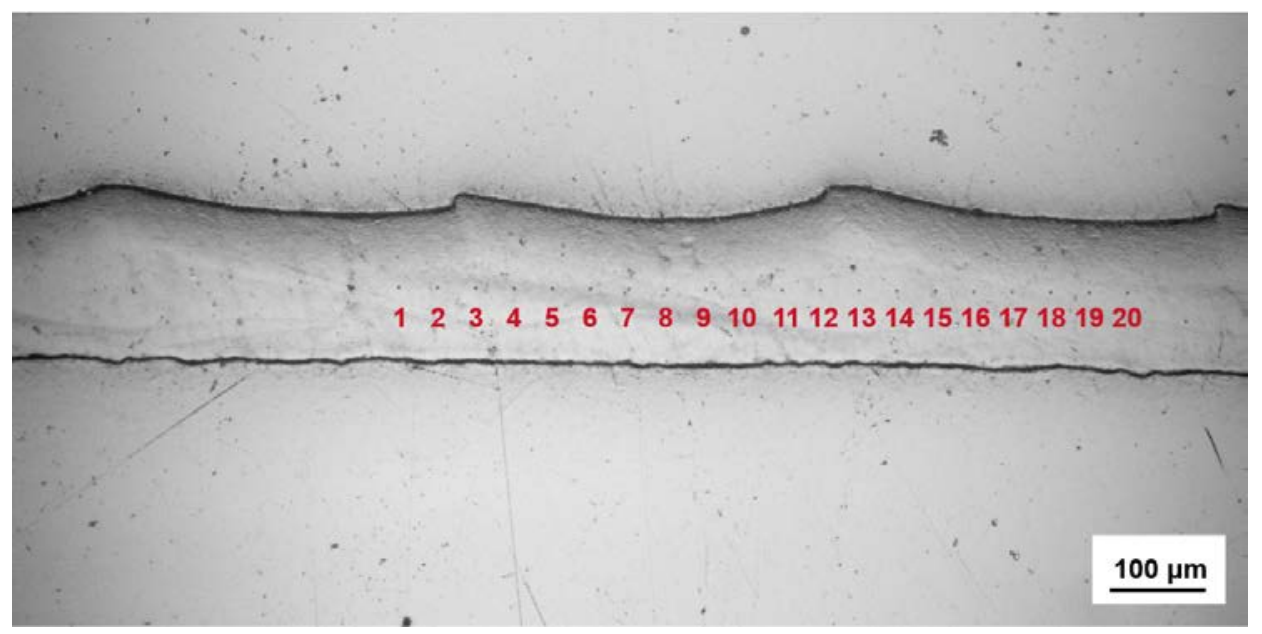

(a)

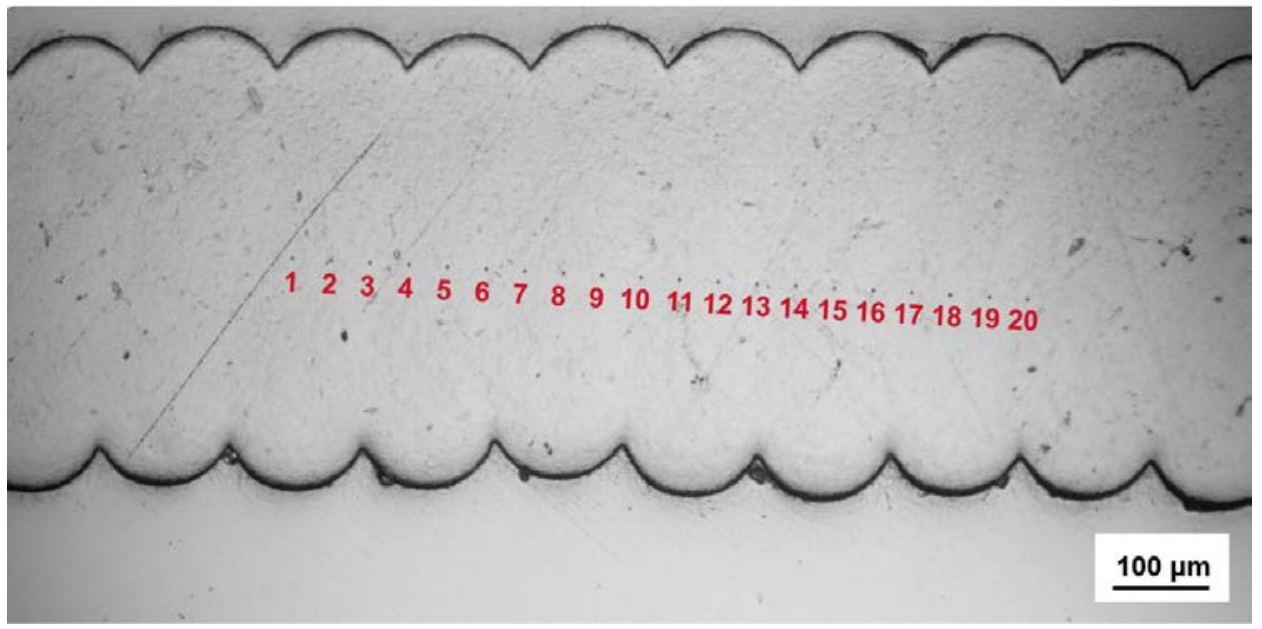

(b) 


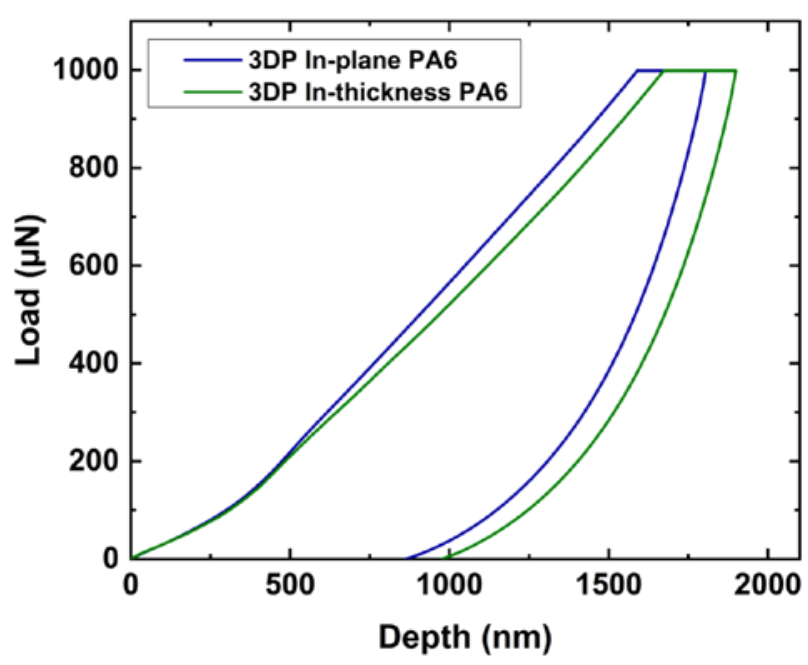

(c)

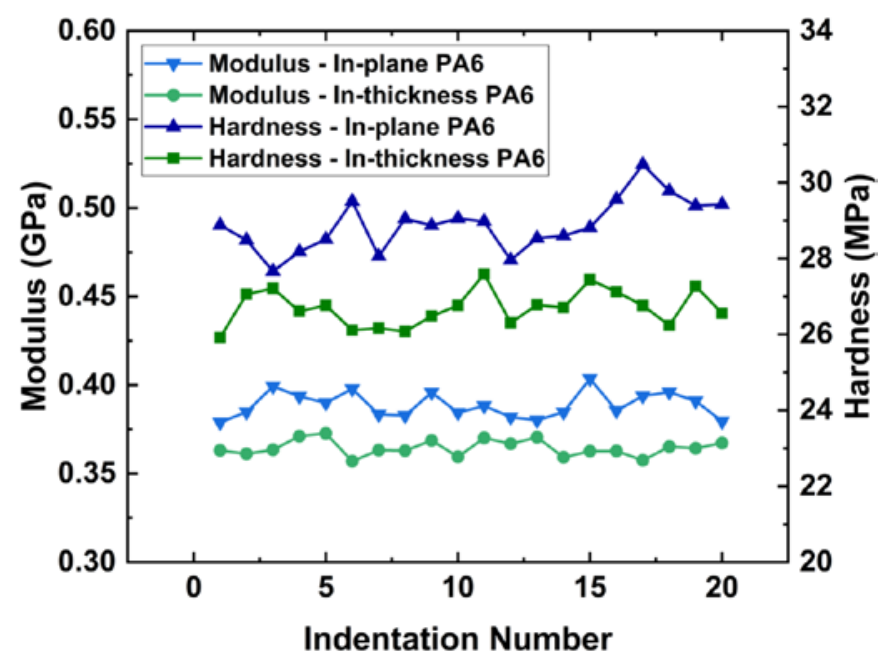

(d)

Figure 15. Nanoindentation test results of the 3D printed PA6 films: (a) micrograph showing indent test areas on a single-layer, 'in-plane’ deposition mode film, (b) micrograph showing indent test areas on a single-filament, ‘in-thickness’ deposition mode film, (c) typical load-depth curves from a single test area and (d) results of modulus versus hardness against the number of the indented point.

\subsection{Summary}

It should be noted that, from all the relevant tests conducted, the extent and quality of the fusion bonding of the 3D printed films via both the 'in-plane' and 'in-thickness' deposition modes were much lower than that attained in the 'in-plane' deposition mode films made using the FFF process but followed by a further $\mathrm{CM}$ process. These observations may be attributed to the relatively low pressure and narrow thermalprocessing window of the FFF process that may not ensure sufficient molecular inter-diffusion has occurred to give a relatively high and reproducible level of intrinsic adhesion [22] between the filaments. Therefore, the fusion bonding formed during a 3D printing method based solely upon the FFF process clearly needs to be improved when fabricating parts for practical applications. Such an improvement can possibly be achieved by optimising the 3D printing parameters. However, such a study is beyond the scope of the research presented in the current paper. Nevertheless, the methods developed in this study do enable the characterisation of the extent and quality of the fusion bonding between individual filaments during the FFF process. Further, as shown by Chen et al. [23], a numerical finite element analysis may be used to model the mechanics of the EWF testing. Such studies might further increase the usefulness of the EWF test (a) in quantifying the fusion 
bonding attained during a 3D printing process and (b) in optimising the FFF process to increase the toughness, and other mechanical properties, of the resulting 3D printed polymeric parts. Finally, it is worth noting that the EWF test methods described in the present study are strictly only applicable for testing ductile polymers but can undoubtedly be extended to ductile polymeric composites and nanocomposites. However, the characterisation of brittle, thin films fabricated by the FFF process requires an alternative test method to be developed.

\section{Conclusions}

In this study, the EWF method was successfully adopted to characterise the quality and extent of the fusion bonding between individual filaments of 3D printed, thin polyamide (PA6) films which were fabricated via both 'in-plane' and 'in-thickness' deposition modes using the fused filament fabrication (FFF) process. The following conclusions can be drawn:

a) Performing EWF tests on 3D printed, thin PA6 films has proven to be a feasible and efficient way to evaluate the extent and quality of fusion bonding between individual filaments of 3D printed polymers. Such tests can provide a valuable insight into the printing quality of the FFF process.

b) The value of the average value of the specific essential work of fracture, $w_{e}$, for 3D printed 'in-plane' deposition mode PA6 films was determined to be $25.7 \mathrm{~kJ} / \mathrm{m}^{2}$, which was $33 \%$ lower than that obtained using the same materials and the same FFF process but now followed by compression moulding (CM). This observation, and the results from the other studies that have been conducted, reveal that insufficient fusion bonding, i.e. intrinsic adhesion, between the filaments of the 3D printed PA6 polymer was developed during the FFF process.

c) The average value of $w_{e}$ for the 3D printed single-filament, 'in-thickness' deposition mode PA6 films was $21.3 \mathrm{~kJ} / \mathrm{m}^{2}$, which was inferior to that developed in the 'in-plane’ deposition mode, with a decrease of $17 \%$ in the value of $w_{e}$, accompanied by a relatively low value of $\mathrm{R}^{2}$, being observed.

d) The value of the coefficient of determination, $R^{2}$, can be an important indicator to quantify the quality, i.e. consistency, of the printing quality using an FFF process, where a high value of $\mathrm{R}^{2}$ indicates a more robust machine and a better printing configuration. 
e) As revealed by the quantitative thermal analysis and nanoindentation tests, a large temperature gradient in the single-filament, 'in-thickness' deposition mode for the FFF process for making the PA6 films contributes to insufficient molecular inter-diffusion between the deposited filaments, which leads to a relatively poor toughness of the 3D printed PA6 film.

\section{Declaration of competing interest}

The authors declare that they have no known competing financial interests or personal relationships that could have appeared to influence the work reported in this paper.

\section{Acknowledgements}

The authors acknowledge the financial support of the Discovery Project (No. DP190102354) from the Australian Research Council. Q.H. He is grateful for the financial support from an Engineering and Information Technologies Research Scholarship at the University of Sydney.

\section{References}

[1] Rodriguez JF, Thomas JP, Renaud JE (2001) Mechanical behavior of acrylonitrile butadiene styrene (ABS) fused deposition materials. Experimental investigation. Rapid Prototyp J 7:148-158. doi: 10.1108/13552540110395547

[2] Chacón JM, Caminero MA, García-Plaza E, Núñez PJ (2017) Additive manufacturing of PLA structures using fused deposition modelling: Effect of process parameters on mechanical properties and their optimal selection. Mater Des 124:143157. doi:10.1016/j.matdes.2017.03.065

[3] Kuznetsov VE, Solonin AN, Urzhumtsev OD, Schilling R, Tavitov AG (2018) Strength of PLA components fabricated with fused deposition technology using a desktop 3D printer as a function of geometrical parameters of the process. Polymers 10:313. doi: 10.3390/polym10030313

[4] Lanzotti A, Grasso M, Staiano G, Martorelli M (2015) The impact of process parameters on mechanical properties of parts fabricated in PLA with an open-source 3-D printer. Rapid Prototyp J 21:604-617. doi:10.1108/Rpj-09-2014-0135

[5] Yang CC, Tian XY, Li DC, Cao Y, Zhao F, Shi CQ (2017) Influence of thermal processing conditions in 3D printing on the crystallinity and mechanical properties of PEEK material. J Mater Process Technol 248:1-7. doi:10.1016/j.jmatprotec.2017.04.027

[6] Sun Q, Rizvi GM, Bellehumeur CT, Gu P (2008) Effect of processing conditions on the bonding quality of FDM polymer filaments. Rapid Prototyp J 14:72-80. doi:10.1108/13552540810862028

[7] Abbott AC, Tandon GP, Bradford RL, Koerner H, Baur JW (2018) Process-structure-property effects on ABS bond strength in fused filament fabrication. Addit Manuf 19:29-38. doi:10.1016/j.addma.2017.11.002

[8] Yin J, Lu CH, Fu JZ, Huang Y, Zheng YX (2018) Interfacial bonding during multi-material fused deposition modeling (FDM) process due to inter-molecular diffusion. Mater Des 150:104-112. doi:10.1016/j.matdes.2018.04.029

[9] Barany T, Czigany T, Karger-Kocsis J (2010) Application of the essential work of fracture (EWF) concept for polymers, related blends and composites: A review. Prog Polym Sci 35:1257-1287. doi:10.1016/j.progpolymsci.2010.07.001

[10] Williams JG, Rink M (2007) The standardisation of the EWF test. Eng Fract Mech 74:1009-1017. doi:10.1016/j.engfracmech.2006.12.017

[11] Mai YW, Cotterell B (1986) On the essential work of ductile fracture in polymers. Int J Fract 32:105-125. doi: 
[12] Karger-Kocsis J, Czigany T, Moskala, EJ (1997) Thickness dependence of work of fracture parameters of an amorphous copolyester. Polymer 38: 4587-4593. doi.org/10.1016/S0032-3861(96)01061-0

[13] Maspoch M, Henault V, Ferrer-Balasa D, Velascob JI, Santana OO (2000) Essential work of fracture on PET films: influence of the thickness and the orientation. Polym Test 19:559-568. doi.org/10.1016/S0142-9418(99)00026-4

[14] Millot C, Fillot LA, Lame O, Sotta P, Seguela R (2015) Assessment of polyamide-6 crystallinity by DSC. J Therm Anal Calorim 122:307-314. doi:10.1007/s10973-015-4670-5

[15] Ma Y, Yokozeki T, Ueda M, Sugahara T, Yang YQ, Hamada H (2017) Effect of polyurethane dispersion as surface treatment for carbon fabrics on mechanical properties of carbon/nylon composites. Compos Sci Technol 151:268-281. doi:10.1016/j.compscitech.2017.08.031

[16] Prashantha K, Schmitt H, Lacrampe MF, Krawczak P (2011) Mechanical behaviour and essential work of fracture of halloysite nanotubes filled polyamide 6 nanocomposites. Compos Sci Technol 71:1859-1866.

doi:10.1016/j.compscitech.2011.08.019

[17] Cotterell B, Reddel JK (1977) The essential work of plane stress ductile fracture. Int J Fract 13:267-277. doi:10.1007/bf00040143

[18] Yamakawa RS, Razzino CA, Correa CA, Hage E (2004) Influence of notching and molding conditions on determination of EWF parameters in polyamide 6. Polym Test 23:195-202. doi:10.1016/S0142-9418(03)00080-1

[19] Gantenbein S, Masania K, Woigk W, Sesseg JPW, Tervoort TA, Studart AR (2018) Three-dimensional printing of hierarchical liquid-crystal-polymer structures. Nature 561:226-230. doi:10.1038/s41586-018-0474-7

[20] Vaes D, Coppens M, Goderis B, Zoetelief W, Van Puyvelde P (2019) Assessment of crystallinity development during fused filament fabrication through fast scanning chip calorimetry. Appl Sci 9:2676. doi: 10.3390/app9132676

[21] Oliver WC, Pharr GM (2004) Measurement of hardness and elastic modulus by instrumented indentation: Advances in understanding and refinements to methodology. J Mater Res 19:3-20. doi: 10.1557/jmr.2004.0002

[22] Kinloch AJ (1987) Adhesion and adhesives: Science and technology. Chapman and Hall, London, UK. ISBN 0-412-27440X

[23] Chen X-H, Mai Y-W, Tong P, Zhang L-C (2000) Numerical simulation of the essential fracture work method. In: Williams JG, Pavan A (eds) Fracture of Polymers, Composites and Adhesives, Second ESIS TC4 Conference on Fracture of Polymers, Composites and Adhesives, Vol 27. European Structural Integrity Society. Elsevier, pp 175-186. doi:10.1016/s15661369(00)80017-5 